University of Wollongong

Research Online

Faculty of Law, Humanities and the Arts Papers (Archive)

Faculty of Arts, Social Sciences \& Humanities

$1-1-2018$

Swarm networks and the design process of a distributed meme warfare campaign

Travis Wall

University of Wollongong, tw590@uowmail.edu.au

Teodor E. Mitew

University of Wollongong, tmitew@uow.edu.au

Follow this and additional works at: https://ro.uow.edu.au/lhapapers

Part of the Arts and Humanities Commons, and the Law Commons

Research Online is the open access institutional repository for the University of Wollongong. For further information contact the UOW Library: research-pubs@uow.edu.au 


\section{Swarm networks and the design process of a distributed meme warfare campaign}

\section{Keywords}

campaign, process, design, networks, warfare, distributed, swarm, meme

\section{Disciplines}

Arts and Humanities | Law

\section{Publication Details}

Wall, T. \& Mitew, T. E. "Swarm networks and the design process of a distributed meme warfare campaign." First Monday 22 .5-7 (2018): 1-33. 


\title{
Swarm networks and the design process of a distributed meme warfare campaign by Travis Wall and Teodor Mitew
}

\begin{abstract}
This paper aims to develop a systemic perspective of the mechanics of an online memetic warfare campaign. The paper uses as its case study the \#DraftOurDaughters campaign, a viral memetic campaign conducted in October 2016 as part of the U.S. presidential election campaign. \#DraftOurDaughters was organised and produced by anonymous members of the Internet board 4chan, and then deployed to wider audiences on platforms such as Reddit, Twitter and Facebook. This process is documented from inception to completion, capturing the swarm like topology of 4chan's /pol/ forum, and the logistics of the swarm's rapid prototyping, coordination, production and dissemination of content. Through examining these phenomena, this paper also provides perspective on the manifestation of collaborative design practice in online participatory spaces.
\end{abstract}

\section{Contents}

Introduction

Meme warfare

Distributed networks, ㅇpen source and swarms

\#DraftOurDaughters

Properties of a memetic warfare campaign

Conclusion

\section{Introduction}

\begin{abstract}
Tactical media, then, would be a kind of filth - an organic process - as compared with the ideological cleanliness of strategic media (the "author"). (...) To preserve its autonomy the tactical medium wants to remain dirty - it can never let itself be surrounded and cleared by strategy, by ideology. It must stay out ahead, drifting before all possible waves, uncertain even of its own trajectory. (...) By another paradox, this uncertainty itself becomes a "principle." It comes to occupy the space of a strategy - and thus to define a strategic space. No "authors" need to be implicated. A messy organic process - involving both reason and unreason - not imposed or categorical - emergent. Shape-shifting. Dangerous and plagued by failures. But not aimless or undirected. In effect - strategic. (Wilson, 1997)
\end{abstract}

The 2016 U.S. presidential elections were surrounded by a vast social media campaign involving the phenomenon of political propaganda deployed across social media on a scale unseen before. October, the final month of the elections, witnessed the \#DraftOurDaughters viral campaign, organised and produced by anonymous members of the Internet board 4chan. Initially, ideas for the campaign began appearing on Twitter and 4chan's politically incorrect themed forum '/pol/'. From there, the campaign quickly gathered momentum across /pol/ and Reddit's 'The_Donald' subreddit. Over the following three days users swarmed to further ideate concepts, rapidly prototyping memetic media objects and deploying them to wider audiences on platforms such as Reddit, Twitter and Facebook.

We believe that the open and visible logistics of this campaign provide an opportunity to map the dynamics of the process and develop a coherent and systemic perspective of the performative mechanics of meme warfare in anonymous online spaces. The \#DraftOurDaughters campaign raises important questions about the nature of online creative collaboration in the construction of media objects, and the way anonymous spaces such as 4chan potentiate and scale up the swarm-like behavior of their user-base. Furthermore, we believe that by observing the mechanics of this campaign we can deduce what enables online spaces such as 4chan to facilitate a collaborative design process where users rapidly prototype ideas and create semantically targeted media.

\section{Meme warfare}

The appearance of Internet memes as political propaganda in periods of political or military conflict is a growing area of research. It is grounded in media studies literature around ideas of participatory media (Jenkins, 2006; Bruns, 2010), as well as viral and spreadable media objects (Jenkins, et al., 2013). Conceptually, the Internet can be described as a "copy machine" space based on a protocol of memetic replication (Kelly, 2008), and Internet memes are indeed emerging as the 
native language of Internet social media. Existing studies of Internet memes generally focus on the replication and spreading of memetic content, both in terms of the production and spread of media objects often existing in many slight variations (Gal, et al., 2015), and the general spread of ideas through online communications (Knobel and Lankshear, 2007).

In this context, memetic warfare has been described as "competition over narrative, ideas, and social control in a socialmedia battlefield" [1]]. Recent case studies of the 2014 Russia-Ukraine conflict by Bradley Wiggins (2016) and the 2014 Israel-Gaza conflict by Chris Rodley (2016) document examples of memetic warfare, where Internet memes are deployed in battles of politically motivated and semantically targeted content across social media as part of a period of broader conflict. The content documented in both studies follows the typical culture of Internet memes, where content creation is driven by remixing and recontextualisation of recognisable popular culture images to create novel ways of carrying and distributing messages (Milner, 2013a). Chris Rodley's study observes political content as "single cultural units which are forwarded widely in their original form", leading to the term "viral agitprop", while Bradley Wiggins' study differentiates by documenting content following a unified message carried out through many variations.

These early scholarly treatments of Internet meme cultures provide the groundwork for exploring the space of memetic warfare. Accordingly, we are interested in the mechanics of targeted and collaborative memetic content production. In 2015, Jeff Giesea called for a conceptual understanding of memetic warfare, positioning it in the realm of information warfare [2]:

One might think of it as a subset of 'information operations' tailored to social media. Information operations involve the collection and dissemination of information to establish a competitive advantage over an opponent. Memetic warfare could also be viewed as a 'digital native' version of psychological warfare, more commonly known as propaganda.

Our aim is to develop a framework for understanding the dynamics of targeted memetic content production as an information warfare operation. There are two key dynamics which we argue are of particular interest in the collaborative production of memetic content. The first dynamic is topologic and involves the network topology according to which actors organise themselves around a project. It is our contention that the spatial affordances of the network's topology determine the performative nature of the distributed content production process. The second dynamic is processual, and has to do with the nature of this content production process. We argue that rapid error discovery and fast feedback loops are emergent functions of a distributed content production process, leading to the incredible fluidity and adaptability of a distributed memetic campaign.

\section{Distributed networks, open source and swarms}

Our framework of topologic and processual dynamics is constructed with concepts native to systems theory (Baran, 1962), the open source movement (Raymond 2001; Robb, 2007), swarming in conflict scenarios (Arquilla and Ronfeldt, 2000; Rheingold, 2002), and approaches to fourth generation warfare (Lind and Thiele, 2015). The behavior of the swarm in response to an identified goal is mapped to concepts of feedback loops (Boyd, 1986), and design process methodology (Dubberly, 2008; Lawson, 2005).

\section{Distributed networks}

First, it is important to establish various digital communication network configurations and their different topological affordances. Paul Baran's 1962 paper "On distributed communications networks" is useful groundwork, establishing three general configuration settings: centralised, where all nodes are linked to a central point; decentralised, where a series of centralised networks are linked; and distributed, where all the nodes are linked without having any central point (Figure 1 ). In practice, most communications networks are a combination of all three [ $\underline{3}]$ and the systems might be best thought of as a spectrum ranging from totally centralised to totally distributed.

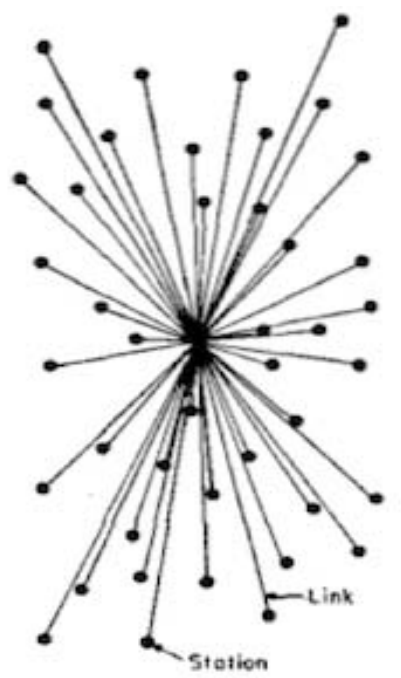

(a)

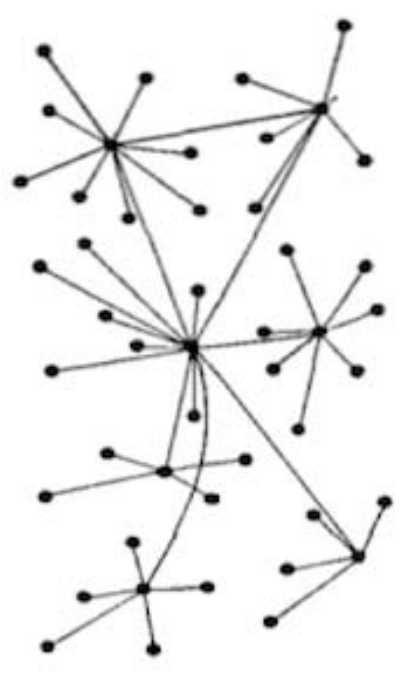

(b)

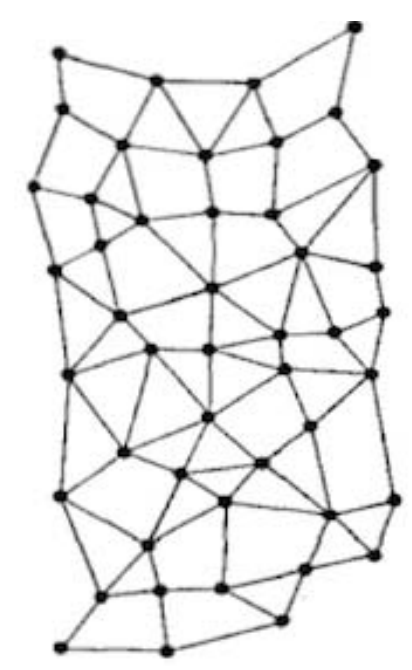

(c)

Fig. 1-(a) Centralized. (b) Decentralized. (c) Distributed networks. 
Figure 1: Paul Baran's (1962) three models of communication network topologies - centralised, decentralised and distributed.

Arguably, Baran's primary interest was in designing communications networks balancing efficiency and survivability. In this case, efficiency refers to the logistics of information flow through the network. Centralised networks operate efficiently because information from the originating node is sent directly to the central hub and then swiftly distributed to the intended recipient. Distributed networks are much less efficient because information bounces throughout the network until it finds its intended recipient, potentially making many redundant moves. Survivability refers to the layout of the network. Centralised networks are significantly more fragile because removing a central node breaks the network of connected nodes [4]. Distributed communications networks are highly resilient, as nodes can be destroyed or removed from the network and it continues to function [ $\underline{5}]$.

The significant advantage of the distributed network topology is its scalability. In 1962 Baran was anticipating the need for a global network of information exchange, in the process observing that at the cost of redundancy a distributed communications network is highly scalable without any major reconfiguration [ㅌ]. Nodes can be simply added or removed from the network without significant conceptual or technical reconfiguration. The key to this, not explicitly mentioned by Baran, is the rise of coordination costs associated with the scaling up of highly efficient networks such as centralised topologies. As more nodes join a network, it can be said to scale up, which in turn increases the costs of coordinating the flow of information between nodes in the network. Crucially, these coordination costs are an inevitable emergent function of any network performing itself into existence against external resistance. As an aside, in this context it has to be emphasised that all nodes of an information network are necessary for its performativity, "if a node is redundant the network drops it" []].

A hierarchical and vertically integrated network, with a central hub, will experience high coordination costs as it scales up simply because of the strain the new nodes will pose on the center of calculation in the network. Importantly, a highly centralised network will be highly efficient in the way in which it processes information - from peripheral nodes to the center - while also incurring prohibitive coordination costs as it scales up beyond a certain size. This however is not the case for a distributed network, which can both scale up easily and maintain low coordination costs as there is no pronounced center of calculation and coordination. This also means that in a distributed topology the entire surface of the network acts as a periphery as there is no designated centre. This is a crucial point for understanding the dynamics of distributed memetic warfare campaigns, and we will return to it below.

The concept of distributed networks is not limited to technical communications networks. Henry Mintzberg's (1979) seminal text on organisation structure The structuring of organisations, and in particular the chapter 'The organisation as a system of flows', uses these concepts to illustrate ways groups organise. He identifies five typically occurring organisation types; simple structure, machine bureaucracy, professional bureaucracy, divisionalised form, and adhocracy, and these structures can be used to describe many typical organisation types, from small technology start-up companies to multinational corporate organisations (Bolman and Deal, 2008).

This set of structures falls into a spectrum closely aligning to Baran's model of network structures. Simple structures resemble a centralised network, where all decision-making in the organisation goes through a small number of people. Divisionalised structures resemble a decentralised network, where organisations divide into smaller centralised networks. Adhocracy structures resemble a distributed network, where people organise and operate without clear hierarchy or mechanisms of centralisation [ㅁ].

\section{Open source}

The mechanics of open source projects provide an excellent illustration of the production logistics in a distributed network, both in terms of organisational topology and information exchange. Open source forms of organisation gained traction in the 1980 s in software development, primarily with university and corporate researchers, as a reaction to the spread of expensive proprietary software with locked and inaccessible source code displacing the heretofore default culture of freely distributed software with source code open for access and modification. The answer to this was a movement of people building their own software, making the source code available to anybody to improve or modify for their own purposes and distributing it across the emerging Internet (Weber, 2004).

The classic text on the mechanics of how people organise around open source projects is Eric Raymond's (2001) The cathedral and the bazaar, which draws from Raymond's experience developing an open source e-mail client and observations on the development of the open source Linux operating system. The cathedral and bazaar are metaphors of network topology contrasts. Raymond describes development of proprietary software as the building of a cathedral which is "carefully crafted by individual wizards or small bands of mages working in splendid isolation, with no beta to be released before its time" []ㅡ. In contrast, open source projects operate in a chaotic style, where methods, materials and ideas are held in common, copied, traded and shared, and the state of things is in a functional but seemingly constant flux [10]. Raymond's observations of open source projects typically resemble an "adhocracy" organisational structure, particularly in the sense that adhocracies are "most often found in conditions of turbulence and rapid change" [11].

As Steven Weber notes, open source is attractive because of the bazaar dynamic of openness and sharing of ideas and materials, but its biggest strength is the way anybody can join or leave the project at any time [12]. This is the function of scalability we discussed above in the context of distributed network topologies. In an open source project, entry to the project is largely unlimited and therefore the project team can scale up or down at any stage as needed without any reconfiguration. Crucially, and this is a fundamental element of understanding the dynamics of distributed memetic warfare campaigns, this function of a distributed network is only valid insofar as it uses an open-source coordination protocol. If that protocol were to change, the network would very quickly fragment or morph into a more centralised topology. In other words, distributed networks have to, axiomatically, adopt Raymond's bazaar model if they want to be able to maintain operations at scale. As an interesting aside, the open source organisational protocol allows change-dependent rapid prototyping and experimentation across the entire network periphery, where the network interfaces with other, often resistant, networks.

\section{Open source insurgency and swarming}

While common examples of open source projects usually revolve around the mechanics of people organising, accessing and working on developing technology, the concept of open source protocol has also been used to describe political projects. 
Open source insurgency is a term developed by U.S. military and political analyst John Robb to describe how political movements operate with the mechanics of an open source project, complete with bazaar-like dynamics and distributed network features of scalability. John Robb's observations come from his experience in military operations in Iraq and the Middle East from 2002-2008, and are fully developed in his book Brave New War (2007) and on his blog Global Guerrillas.

Participating in an AMA (ask me anything) session on Reddit, John Robb (2016) argued that an open source insurgency revolves around the notion of a "plausible promise", where the primary requirement of the movement is "a shared goal that is broad enough to interpret according to one's own needs". In Brave New War, Robb describes the open source insurgency in Iraq and the Middle East as made up of at least seventy groups from a variety of religious backgrounds and business cartels, all sharing the goal of resisting U.S. occupation of Iraq for different reasons [13]. These groups operate across all fronts, from physical combat to online media [14]. The concept of open source applies to the insurgency in the sense that the entire project of resisting the U.S. occupation has a completely open operational protocol, meaning "the tactics, weapons, strategies, target selection, planning methods, and team dynamics are all open to community improvement" [15] as long as it fits the plausible promise end goal. In Henry Mintzberg's terms, it operates as an adhocracy, as there is no fixed central command and control of the movement. Here, as with Paul Baran's distributed network, nodes can join at any time and the network scales without any organisational reconfiguration or increased coordination costs.

John Robb also stresses how open source insurgencies appear in many different locations to attack facets of the network making up the opposition, seemingly operating to create system disruption and cascades of failure rather than swift blows of single impact [16]. Because of the absence of centralised command and the open nature of the project, actors can join the insurgency at will, and test any strategy, operation or tactic, therefore increasing the unpredictability of the insurgency at scale. The scalable and unpredictable nature of the insurgency can manifest itself in many forms across the entire periphery of the distributed network, from direct force engagements or attacks on critical infrastructure, to individuals performing terrorist acts in Western cities, as well as attacks across media under the same open source dynamics.

The democratised nature of participatory media extends the concept of scalability, as anybody with Internet access and basic tools can create and distribute their own media for the campaign, essentially becoming a node of the network performing the plausible promise. This generates conditions resembling fourth generation warfare, where lines between combatants and civilians are blurred in warfare conducted across a comprehensive front combining political, economic, social and physical combat (Lind and Thiele, 2015). Studies by Chris Rodley (2016) and Bradley Wiggins (2016) become critical foundational pieces in building a background on memetic warfare, as they point toward the propaganda component of a larger network taking part in combat across these fronts.

While John Robb assembles a holistic documentation of the open source distributed network operating on multiple fronts under the conditions of fourth generation warfare, the key mechanic of how people gather around these projects has been thoroughly explored by John Arquilla and David Ronfeldt (2000) in their Swarming and the Future of Conflict. In similar terms to Robb, Arquilla and Ronfeldt look at various insurgency movements across a spectrum of operations, from guerrilla armies engaging in physical combat to political activist groups. Arquilla and Ronfeldt examine how these insurgencies "wreak havoc" on established and highly coordinated institutions, systems and military operations seemingly vastly superior in terms of resources [17]. They describe the key mechanic to the success of these insurgencies as swarming. The concern of Arquilla and Ronfeldt is how this mode of conflict specifically attacks "mass-oriented, industrial-age way of war" and inverts it into a weakness, calling for a rethinking of established military tactics [18]. Early application of this idea in the space of communications technologies has been explored by Howard Rheingold (2002), pointing to Arquilla and Ronfeldt to describe how people swarm to exchange text based information via mobile phones to create social or political disruption [19].

Arquilla and Ronfeldt describe swarming tactics as existing in great variation, where a broader insurgency might feature all possible combinations of these attacks. Hive-like structures typically nest and then deploy omnidirectional attacks to overwhelm a target (like guerrilla movements or activist groups), small mobile units may band together into packs (like soccer hooligans) or opportunistic mobbing where individuals take part for their own personal gain coincidentally aligning with others (like paparazzi) [20]. These variations can only work under a distributed network dynamic, mirroring the open source protocol, as the lack of a centralised coordination mechanism affords wide variance in operation in a freely scalable network. The plausible promise then acts like a glue banding these variants into a messy leaderless swarm of autonomous or semi-autonomous units churning chaotically towards a unified goal. Arquilla and Ronfeldt describe the primary modality of a swarm attack as "sustainable pulsing" - able to attack common targets from multiple perspectives simultaneously, and scaling the attacks on aspects of the enemy network as required [21]].

It is of primary importance to think of this dynamic in terms of coordination costs, as the swarm pulses in whatever direction is needed, and with whatever intensity, without incurring any major costs to reconfigure its organisational topology. Arquilla and Ronfeldt articulate the design of a swarm as follows [22]:

- Many small, dispersed, Internetted maneuver units

- All-service coordination for mixing and matching

- Both stand-off and close-in capabilities

- Integrated surveillance, sensors, C4I for " topsight"

- Aim: " sustainable pulsing" of force and/or fire

- Result: amorphous but coordinated way to strike from all directions - stealthy ubiquity, no " front"

- Tenet: centralized strategy, decentralized tactics, distributed formations and logistics

Interestingly, commenting on the 2016 U.S. presidential elections, John Robb described the movement catalysing around Donald Trump' s candidacy as an open source insurgency. In this context, the shared goal and plausible promise that the open source insurgency swarmed around was removal of the existing network of political establishment and media. There are many groups banding together around this goal, from business cartels, to alternative media organisations, to online activists interested in disrupting systems for their own entertainment. This fits with Arquilla and Ronfeldt' s description of the variations of bands within a swarm. It has been argued that Donald Trump's well documented lack of consistency and coherence on policy positions has effectively helped him operate as a shell, open enough for many to swarm around, interpret or use, which then allows many groups to become part of the swarm (Robb, 2016).

Following Arquilla and Ronfeldt's model, we can observe that the resulting open source insurgency creates sustainable pulse areas surrounding the nexus of political establishment, legacy media and Democratic Party operations, creating cascades of failures. In effect, Donald Trump's campaign network has been able to disrupt the political system from the inside by benefitting from the swarming waves of memetic content attacks across social media.

Feedback loops 
Whatever a network's topology, the speed with which it processes external information inputs and reacts to them is a fundamental function of its coordination costs. This feedback loop is particularly interesting in swarms, because their fully distributed topology allows them to process information inputs locally and react to them with much lower time-delay than more centralised networks. In the context of this argument it is of particular importance to underline the role of fast feedback loops in swarm networks, because the speed of circulation of feedback allows for coordination and control to be performed both locally and at a distance, without altering the network's topology (Callon and Law, 2004). Feedback loops typically occur at both the individual and organisation levels. Individuals cycle through the loop at their own pace, while the organisation also cycles as a whole [23]. At the level of the individual cycling through feedback loops, the lack of hierarchical centralisation and control allows free exploration of ideas, generating a flourishing of creativity and new directions [24].

John Robb points to a specific example of how the open source insurgency uses this mechanic in an example of the rapid

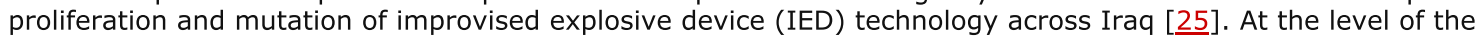
organisation, many nodes operating autonomously mean ideas and experiments within the organisation can be widely varied, which in turn generates a myriad of possible directions across the insurgency. This means that the network is less prone to stagnancy in its operations, and can rapidly generate new ideas and technologies. This is the primary strength of an adhocracy [26], and echoes Eric Raymond' s idea that bugs in software "turn shallow pretty quickly when exposed to a thousand eager co-developers pounding on every single new release" [27].

In the case of rapidly adapting swarms, a feedback loop of decision-making is best modelled by the notion of an OODA loop (see Figure 2) - a feedback decision cycle of observing, orienting, deciding and acting first developed by military strategist John Boyd (1986) for application in decision-making during combat operations. In network terms, an open source insurgency is a distributed network of nodes engaged in their own autonomous OODA loops as they tinker with the campaign. This can happen at multiple levels, from longer term narrative arcs to rapid response to events. When operating under conditions of fourth generation warfare, the production of media content is open to swarming. The OODA loop concept also maps well to design process literature focusing on use of feedback loops in developing design artefacts (Dubberly, 2005; Lawson, 2005).

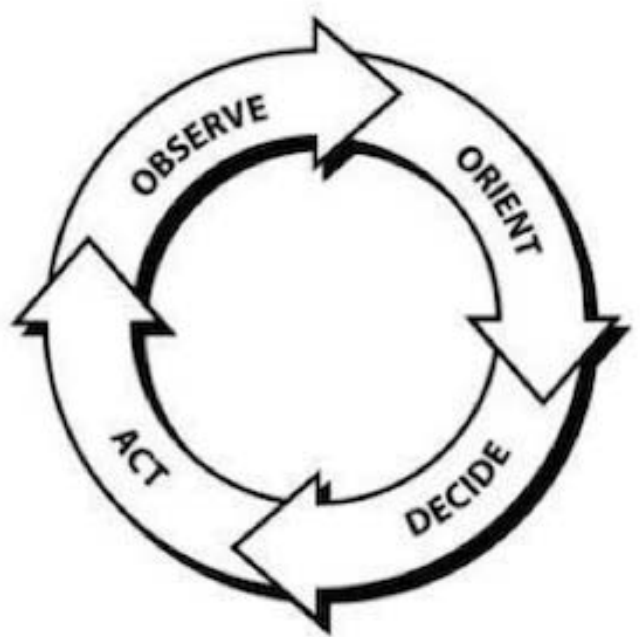

Figure 2: John Boyd's OODA loop.

In the observation stage, information about the situation surrounding the network is gathered. In design process terms this is the phase of research undertaken to develop an understanding of the situation [28]. Orienting is analysis of the information gathered, which maps to the design process phase where a problem is defined, and sets up how the situation is framed [29]. Deciding is hypothesising some actions would produce a particular outcome, which resembles the typical ideation phase of design process modelling [30]. Acting is the stage of performing the hypothesised action in the scenario, which is modelled in design process literature as prototyping, testing, or implementation [31]. This bases the OODA loop in a continuous cycle of feedback and response based in a process of analysis, synthesis and evaluation [32].

\section{\#DraftOurDaughters}

Equipped with the conceptual apparatus discussed above, we are now able to unpack the logistics of the \#DraftOurDaughters campaign, which took place on 4chan's politically incorrect themed forum /pol/ in late October 2016 in the lead up to the U.S. election on 8 November. 4chan users leveraged growing concern over Hillary Clinton's complex relationship with Russia (Hains, 2016) to create a political media campaign to highlight these issues. A small amount of images resembling official Hillary Clinton campaign material appeared on Twitter, and featured typical Hillary Clinton supporters enthusiastic about the possibility of being drafted into combat with Russia. These images were posted on /pol/ as prototypes of a possible memetic warfare campaign, instigating a swarm of users producing content for the project. Forum users rapidly prototyped media for the campaign, with the swarm massively scaling to produce an enormous amount of coordinated, semiotically targeted content. 
4chan

4chan.org is an Internet forum in the format of an image board with a small but growing research interest. 4chan.org features 70 separate boards organised into a diverse set of topics from anime to fitness (Milner, 2013b). It has several key features distinguishing it from many other social media platforms (Knuttila, 2011; Hine, et al., 2017):

- Users can post in threads with a single image, just text or a combination of both. URLs can be posted.

- Users do not need a 4chan account to view 4chan content.

- There are no accounts, no post history and no direct messaging. Users can attach a name to their post, but rarely do (most users appear under the same screen name as "anonymous"). Users can also use an optional identifier known as a "tripcode" to identify themselves as the same author appearing across threads, but this is rare and often frowned upon on /pol/ as a breach of the protocol of anonymity.

- Each post has a unique ID code associated with the IP address of the user specific to each thread.

- Some boards, such as /pol/, feature country flags tied to the IP address of the user, and users have the option of overriding this flag with a selection of flags of non-state organisations and political positions.

- Users can reply directly to a post or multiple posts at a time, but there is no layered threaded conversation.

- There is no mechanism for feedback - no upvoting or downvoting, sharing posts, favoring posts or any other methods of feedback commonly found on social media.

- Each board can hold a limited number of threads in the catalogue.

The last feature needs further elaboration as it contributes directly to the ephemerality of content posted on /pol/, which in turn leads to an ethos of rapid prototyping and an aesthetics of radical experimentation. As each 4chan board is limited in terms of the number of threads it can support at any one time, threads are moved to the top of the catalogue when they are created or receive new posts. This feature also serves to prevent power law distributions where a few popular threads would end up dominating the board. Instead, threads reach a post limit, slide off the catalogue and are closed, to be permanently deleted soon after. In other words, this feature generates a dynamic prioritising new content (Hine, et al., 2017). In practical terms, threads on popular and active boards such as /pol/ may only survive for a few hours, given the large numbers of posters.

4chan has drawn the attention of researchers from a range of perspectives. It has been identified as a significant driver of Internet culture where many popular Internet memes and chunks of Internet culture originate (Hine, et al., 2017). Attention to 4chan also comes from the perspective of online trolling, which is a significant part of the culture of 4chan (Phillips, 2013; Milner, 2013b; Massanari, 2017). Milner and Massanari also trace this culture bleeding from 4chan to other social media platforms, specifically Reddit and Twitter. Most of this research looks at 4chan's /b/ board, which is a board for random posting (Phillips, 2013; Nissenbaum and Shifman, 2017), with research on other boards and particularly the /pol/ board still emerging (Hine, et al., 2017).

4chan has been described as a "meme factory" (Herwig, 2011), indicating it is a highly creative space of content production. A 2017 study of 4chan's /pol/ board by Hine, et al. found that over a two and a half month period leading up to the 2016 US election (30 June to 12 September), over two million images were posted. A significant amount of original content was observed, with a massive long tail distribution. Half of the images posted did not appear anywhere on 4chan outside of $/ \mathrm{pol} /, 70$ percent of these images were only posted once and 95 percent of images are posted no more than five times. The average size of images posted was $321 \mathrm{~KB}[\underline{33}]$.

From these general configurations, we can see the manifestation of a distributed network in the \#DraftOurDaughters campaign. 4chan's simple mechanics facilitate this, as the lack of accounts eliminates the need for an organisational structure around usernames or social capital build-up around accounts. Social capital build-up is also combated via thread deletion after a short period of inactivity. Even if a sequel thread is created, all users are assigned new user IDs in the new thread unless a tripcode is used, which is unusual [34]. The mechanics of discussion on 4chan also resist centralisation of subject matter, because nesting or threading of comments is very difficult. 4chan also has a very low barrier to entry, with no account or approval needed to post. In other words, the 4chan board creates an environment of radical decentralisation, with the network of contributors distributed geographically as well as platform-wise.

The following images exemplify the sequence of events of the \#DraftOurDaughters campaign. Documentation of users swarming around the project on 4chan, and the bleeding of the campaign over to Reddit and Twitter, evidences the topologic nature of memetic warfare. Documentation of conversations of the users captures the processual aspects of memetic warfare, through the ideation, rapid prototyping, coordinating, producing and spreading of content by the users.

\section{Background}

The setting of the \#DraftOurDaughters campaign is the 2016 U.S. presidential elections. The Draft America's Daughters Act of 2016 was passed, amending "the Military Selective Service Act to extend the registration and conscription requirements of the Selective Service System to all U.S. citizens and residents between the ages of 18 and 26. (Currently such provisions apply only to men)" (U.S. Congress, 2016). This would allow women to be conscripted to military service. There were also various media pieces published online developing a narrative around concern over Hillary Clinton's complex relationship with Russia, and speculation over the possibility of military conflict with Russia under a Hillary Clinton Presidency. These were most prominent in the campaign of the Green Party candidate Jill Stein (Harris, 2016).

\section{First appearances}

On 26-27 October 2016, a small amount of images of what appeared to be Hillary Clinton campaign material featuring people enthusiastic about being drafted for military service in a war with Russia appeared on Twitter. The images use typical Hillary Clinton campaign visual language, featuring the Hillary Clinton campaign branding and people from typical Hillary Clinton campaign images. The images appeared to gain a small amount of traction on Twitter. 


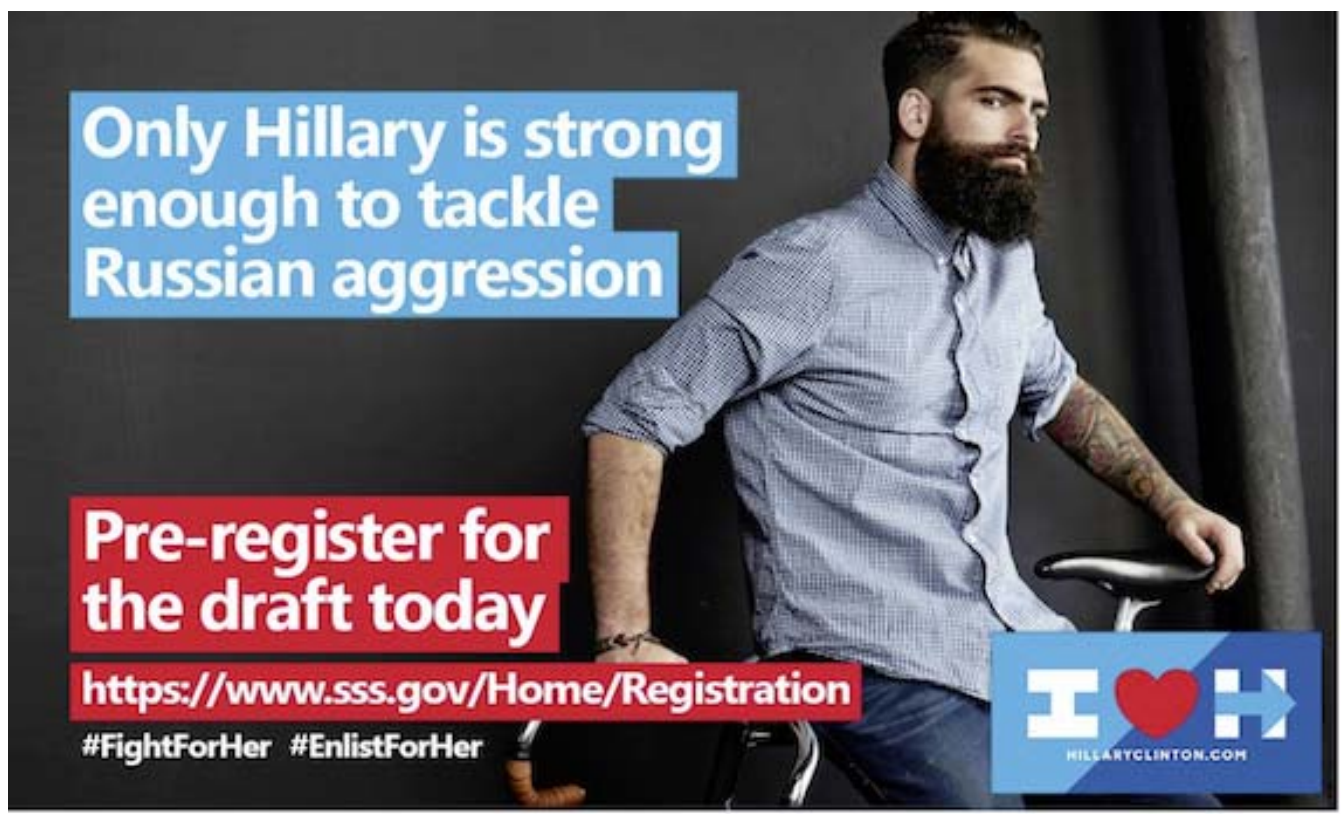

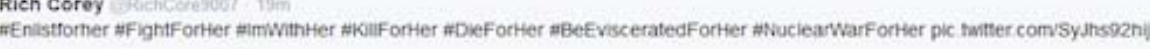
क $2732 \quad \nabla$ a $32 \quad \ldots$

Figure 3: Early campaign content distributed on Twitter. Tweets archived at http://archive.is/FWAE3.

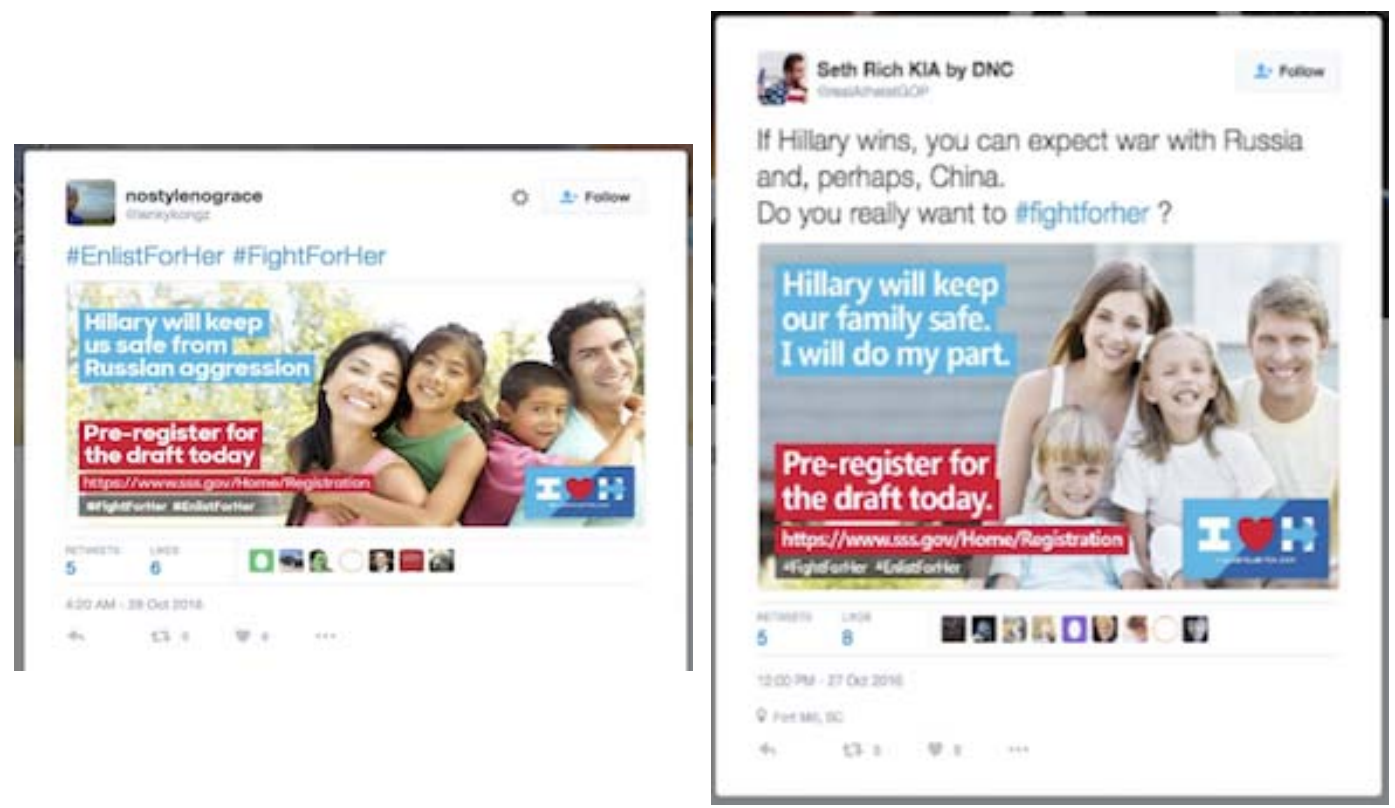

Figure 4: Further examples of early campaign content distributed on Twitter. Tweets archived at http://archive.is/WNRV2 and http://archive.is/Blbzs.

\section{4chan's /pol/ community is mobilized}

The images were posted on 4chan's /pol/ board in two threads revealing the images were not official Hillary Clinton campaign material, but were created by people opposing Hillary Clinton who were using sockpuppet accounts. The traction gained by the images on Twitter was used by 4 chan users as validation of the concept, identifying it as possibly useful for a memetic campaign against the Hillary Clinton campaign. 


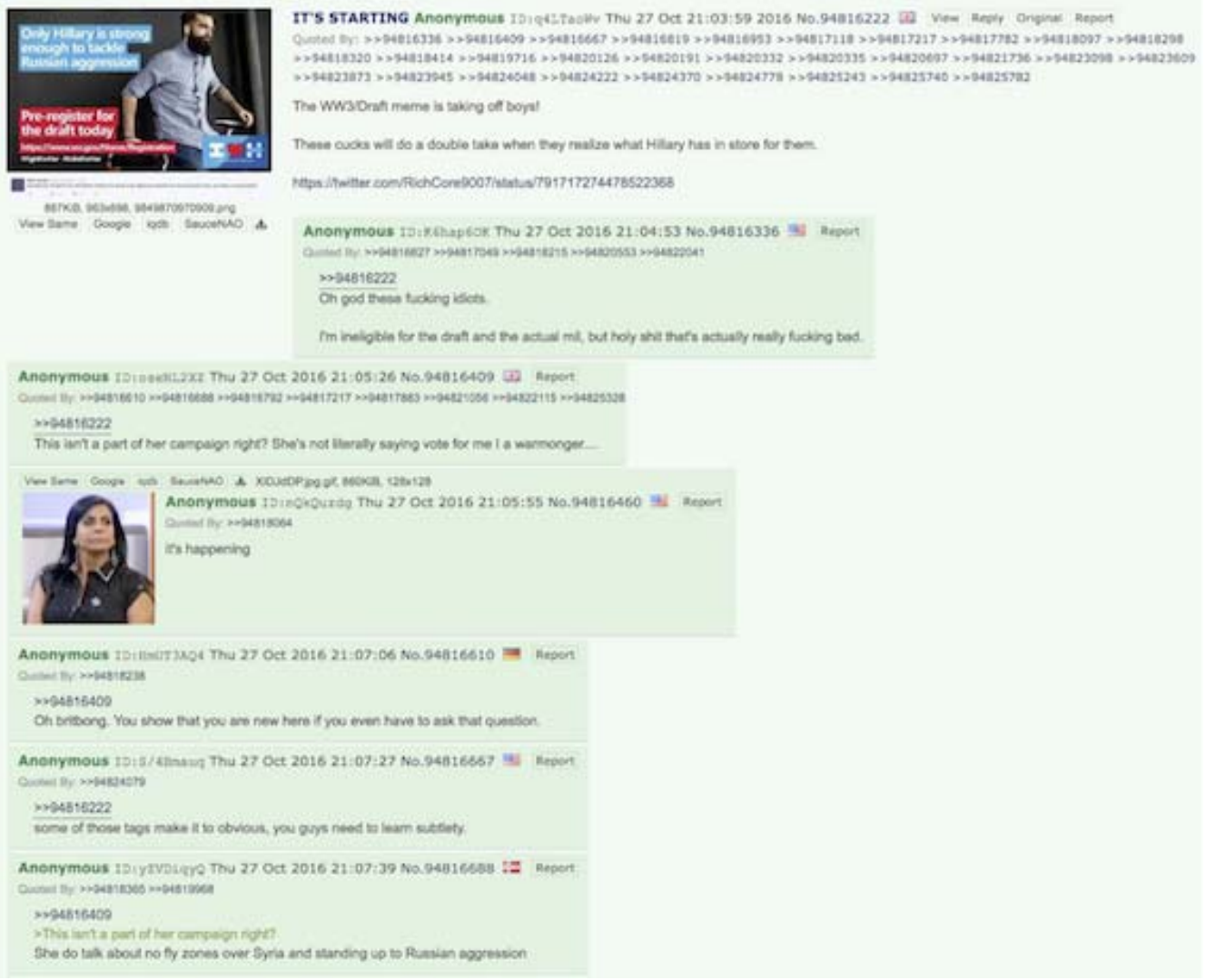

Figure 5: Example of an initial campaign thread on 4chan's /pol/ forum instigating the swarm around the campaign. Full thread archived at http://archive.is/EjgSF.

Note: Larger version of figure available here. 
Send your daughters to war, for equality! \#ImWithHer \#WhySheRuns \#enlistforher \#fightforher \#herfightmyfight \#takeonputin \#worldwar3 \#Trump

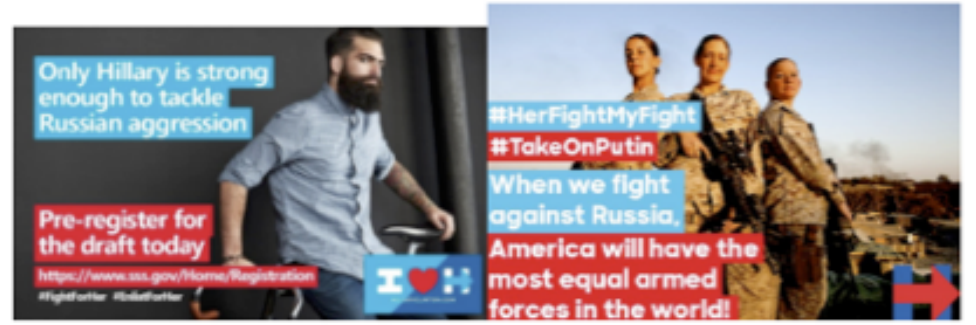

\section{RETWEETS LIKES

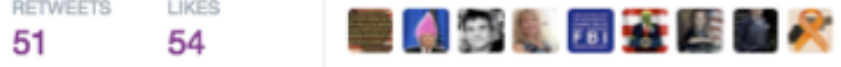

2:15 PM - 27 Oct 2016

4 $\quad$ ᄂ7 $51 \quad 0 \quad 54 \quad \ldots$

67

Keen Gaze KeenGaze - Oct 27

Gnotadroneirl @mybodyGG ok, in all seriousness, IS THIS FUCKING REAL?

a $\quad 12$

deplorable Kimba eKimba212Jupiter - Oct 27

@KeenGaze @notadroneirl @mybodyGG Yes, b/c if Hilda becomes C in C, voluntary enlistment will end, hence, draft. + women. The time is here.

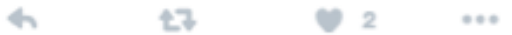

The Ronin ${ }^{\mathrm{TM}}$ eLCMee24 - Oct 27

Qnotadroneirl @magnifier661 they did beg for it so out the feminist up front to shield the better soldiers.

h 27

Figure 6: Early campaign content distributed on Twitter. Tweets archived at http://archive.is/fpwRN.

\section{\#DraftOurDaughters emerges}

In these two threads on /pol/, the topologic nature of memetic warfare manifests itself as a clear swarm appears to explore the concept. The general campaign narrative was established, where users work to the general concept of exploiting the narrative of the Hillary Clinton campaign and the narrative and information available around concern of war with Russia under a Hillary Clinton presidency - "send your daughters to war, for equality!" (Figure 6). In both these threads, the processual dynamics of memetic warfare appear as members of the community make more images exploring the concept, openly experimenting with images, tag lines, hashtags and general tone. Images were simultaneously deployed to Twitter as they were posted to /pol/, a pattern evident in Figure 7 (See Appendix, Figures $\underline{9}, \underline{10}$ for further examples).

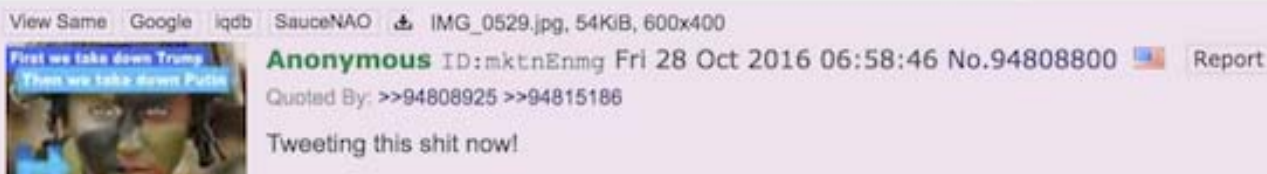




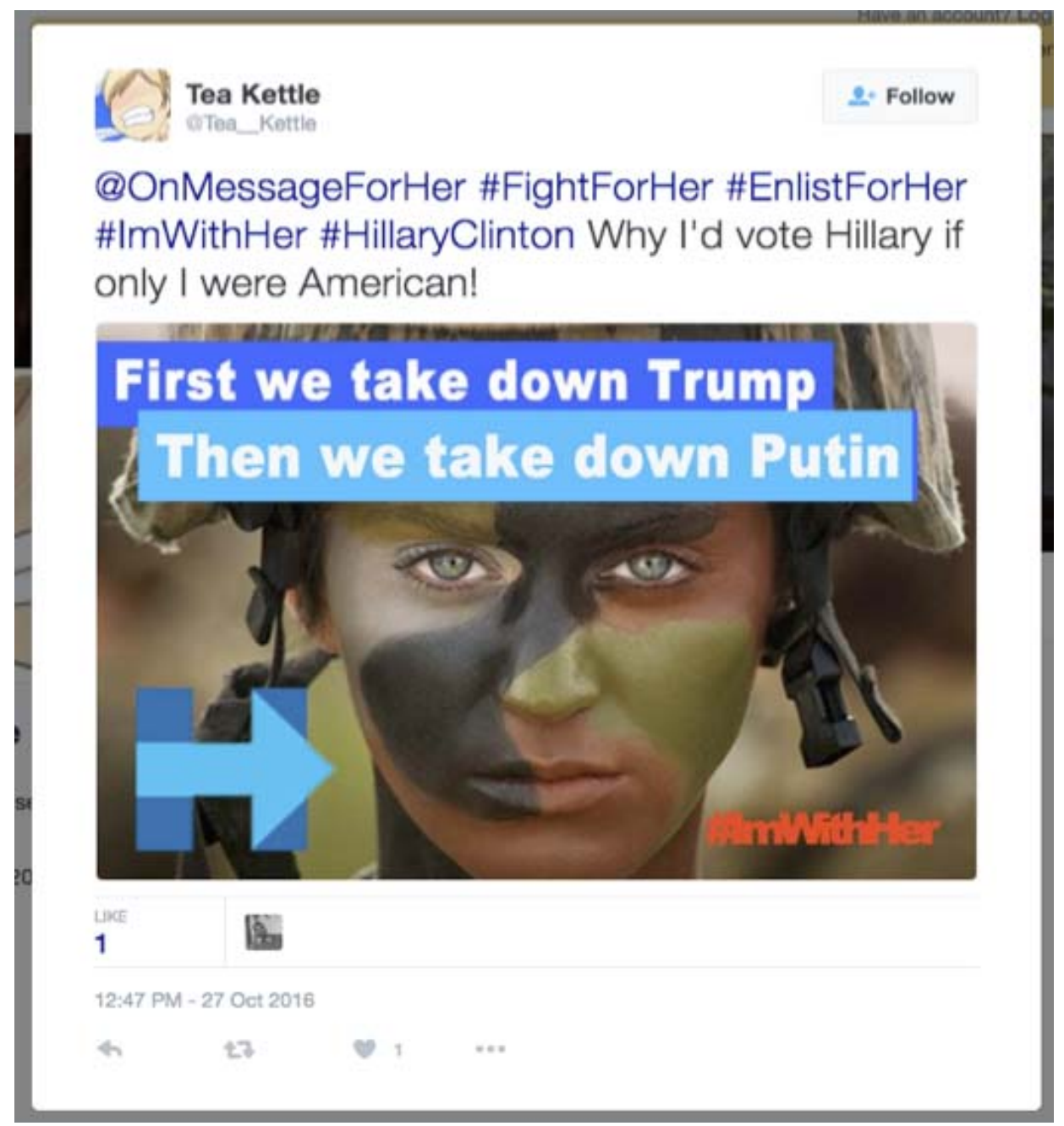

Figure 7: A /pol/ user prototyping content and deploying to Twitter. Post archived at http://archive.4plebs.org/pol/thread/94805438/\#94808800. Tweet archived at http://archive.is/q23f9.

\section{Organising and production of memes}

It is through these threads that the \#DraftOurDaughters campaign emerged. All facets of the campaign were simultaneously developed in public, where users ideate, rapidly prototype and respond to content developed. A collection of screenshots capturing samples of this process is documented in the Appendix below. In these early segments of the process, the intensity of the swarm's engagement with the attack vector can be seen to noticeably rise as indicated by the shortening time intervals between posts as the threads grow (See Appendix, Figures 7-19). Crucially, the rising intensity of swarm engagement can be observed in terms of the shortening of the time intervals of OODA feedback loops, where new targeted memetic content is posted, commented on, and reiterated (See Appendix, Figures 14-19).

The initial concept was refined to a consistent style guide of graphics and images where the Hillary Clinton campaign brand was effectively open sourced by users who extracted the graphic fonts from the Hillary Clinton campaign Web site (See Appendix, Figure 11). The concept for the memetic warfare campaign was then further explored and refined (See Appendix, Figure 12), with multiple slogans developed through a process of rapid prototyping and testing (See Appendix, Figure 13). Notably, users experimented with image and slogan compositions through OODA feedback loops, with the swarm acting as an early test ground closing the loop (See Appendix, Figures 14-18). The same process was used to ideate multiple hashtags for the campaign, with \#DraftOurDaughters emerging as the most popular (See Appendix, Figure 19). The images were also posted to Reddit on $/ r$ /the_donald subreddit, where a similar distributed production process was instigated (Figure 21).

Following the swarming around these initial catalysing threads on /pol/, the swarm systematized its production process by creating discussion threads uniformly titled "/st/ stronger together", where production of the targeted memetic objects continued ( 27 threads). Reminiscent of the swarm pulse attack mechanics described above, an image style guide and template could be observed to stabilise through replication in each new /st/ thread. A detailed set of instructions around fonts, colors, image style and deployment technique developed, and spread throughout the "/st/ stronger together" threads and $/ r$ the_Donald.

\section{Campaign content generated}




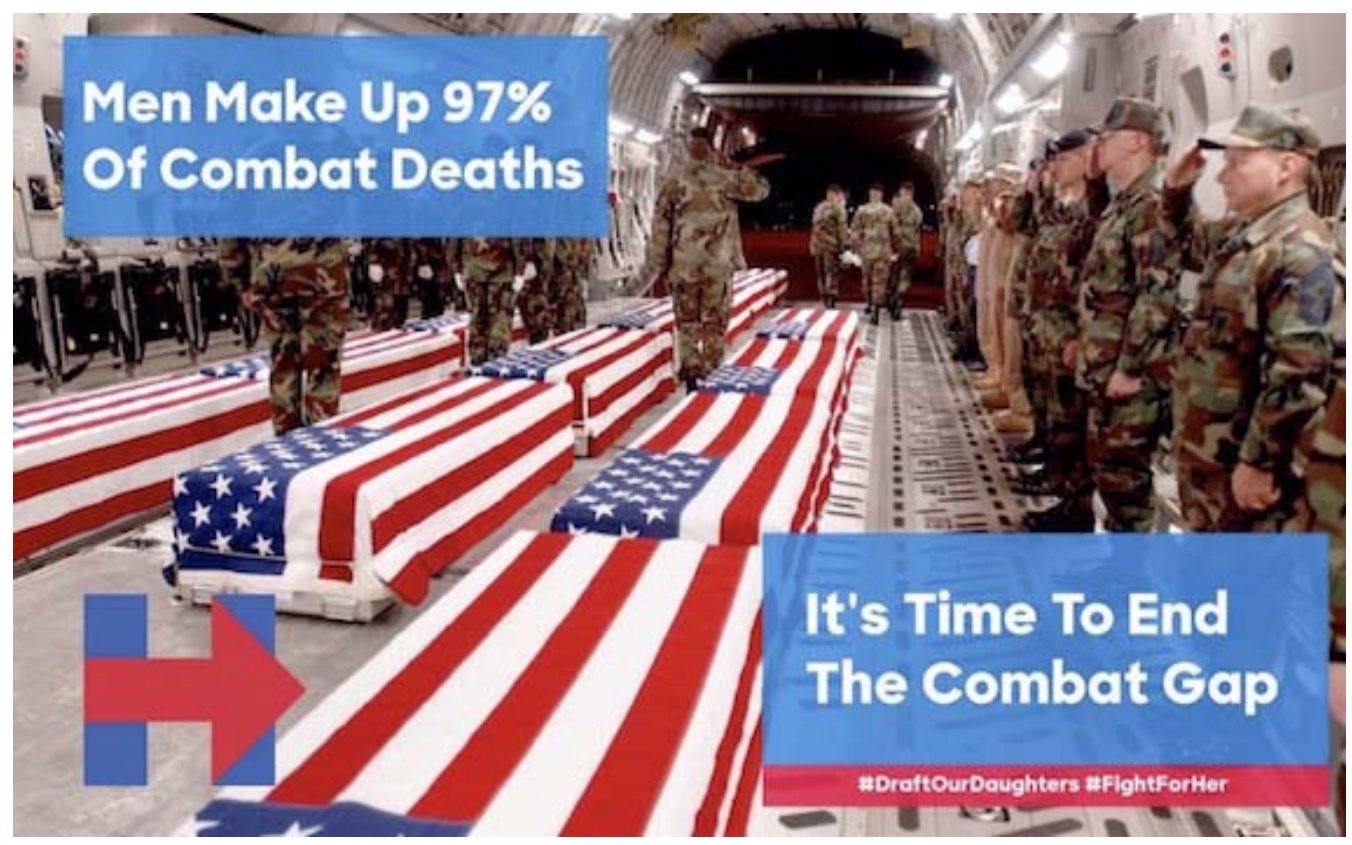

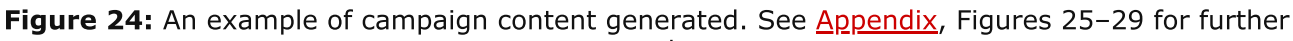
examples.

As can be observed in the Appendix, the fast OODA loop of the swarm led to a continuous refinement of the visual quality of the memetic warfare objects being produced, as well as to the rapid prototyping of new ideas and vectors of attack. Figures 24-29 document a sample of the images produced and deployed in the campaign. With /pol/ acting as the primary swarm space, and / $r$ /the_Donald as its extension, Twitter became a primary distribution platform for the content, with the \#DraftOurDaughters hashtag trending (See Appendix, Figure 30).

The content generated confusion among legitimate Twitter users, with sockpuppet accounts boosting the perception of confusion and falsifying sentiment, therefore adding another vector the the memetic warfare campaign (See Appendix, Figure 31). Interestingly, as the swarm subsided it seemed to generate an ad hoc process of self-reflection, with users attempting to systematize the lessons learned from the process, and ideating a new set of memetic attack vectors (See Appendix, Figures 32, 33).

\section{Properties of a memetic warfare campaign}

Based on the integrated topologic and processual dynamics of targeted memetic content production we developed above, as well as our observations from the \#DraftOurDaughters case study, below we generalize a framework of key components instrumental in meme warfare operations. As we argued above, the topologic dynamic of memetic warfare captures the fundamental importance of distributed networks in allowing rapid scalability without impacting network coordination costs, while the processual dynamic captures the fundamental importance of fast OODA loops when the swarm is engaged in an attack vector at scale.

Given the presence of topologic and processual dynamics, we think the key components instrumental in meme warfare campaigns are public operations, open source protocol, anonymous participants and decision-making through memetics. Together, these components generate a framework featuring a distributed topology of operation, open source project dynamics, swarm features of an open source insurgency, and the performance of rapid feedback loops. Importantly, this framework can be successfully mapped to the findings of previous studies of memetic warfare, notably Bradley Wiggins' (2016) documentation of content around the Russia-Ukraine conflict and Chris Rodley's (2016) documentation of content around the Israel-Gaza conflict. In addition, our framework provides an insight into the kinds of platform affordances that facilitate a successful memetic warfare campaign.

\section{Public operations}

Public visibility of the campaign is critical to the process, both topologically and processually. Topologically, operating openly and in public allows the swarm to massively scale its content generation, as there is no cost of entry and the feedback loops are extremely fast. In addition, as the swarm is always already operating in public, its entire network is in effect a periphery and fully engaged with a target public. Processually, a swarming open source insurgency functions on the principle that groups are constantly able to observe and copy what other groups are doing, taking ideas and adding their own iterations on top of them (Robb, 2007). In \#DraftOurDaughters, we see a similar dynamic in media production and deployment facilitated by the 4chan platform:

- Any individual or group interested in attacking the Hillary Clinton campaign can contribute in any capacity, from starting the project to contributing a single wording suggestion or image. Lack of accounts on the 4chan platform allows effortless scaling of the swarm around the project, with groups distributed across a wide variety of other platforms able to observe, orient, decide and act directly based on ideas generated on /pol/. 
- All users can see what is deployed by all other users, exposing the feedback loops of individual nodes to the rest of the network. The discovery of successful attack vectors is always already communal and immediately replicated across the swarm surface.

- When a successful attack vector is discovered, users swarm around it and operate under open source production to build on top of it. The visibility of this swarming and building indicates to all users a vector with high potential for resonance has been discovered, which then leads to rapid scaling up of the work on that vector.

This dynamic can be observed in other cases of memetic warfare, suggesting that any platform operating primarily as a space for public conversation is a possible facilitator of the swarming dynamic key to a memetic warfare campaign. 4chan, Twitter and Reddit are key examples as evidenced by previous studies on swarming and campaign content creation. In Rodley's documentation of content around the Israel-Gaza conflict, swarms of content creators are observed gathering around the public conversation about the conflict on Twitter, injecting content directly into conversation. Wiggins' documentation of content around the Russia-Ukraine conflict also focuses on Twitter, which is used to inject content into the public conversation. In the case of Wiggins' documentation, the content originally appeared on the "Russian internet" [35] and was deployed on Twitter, reaching wider audiences in a process similar to \#DraftOurDaughters. A similar occurrence of users swarming around a public conversation is found in Massanari's (2017) study of how the Reddit platform facilitated users swarming around a social issue and creating campaign content.

\section{Open source protocol}

An open source protocol of memetic content production builds on the processual dynamic, and is crucial in terms of the iterative nature of the creative process of a meme warfare campaign. Any digital media object is subject to open source properties simply because of the ease with which it can be copied, remixed and redistributed. Digital images are particularly potent, as they can be used as frames carrying complex semantic payloads, while being easy to produce, remix and distribute. The technical barrier to image production entry is very low, aiding distributed topologic dynamics through the ease with which users can join the swarm. Processually, digital images fit a profile of fast, inexpensive, simple, tiny (FIST) project properties observed by U.S. Air Force Lt. Col. Dan Ward as optimal for innovation. FIST generates conditions of fast feedback loops, rapid iteration and quick emergence of successful strategies and technical innovation (Ward, 2011). \#DraftOurDaughters is a key example of the importance of this component of the framework:

- Swarming users treat all content as open source, picking up any existing content with little regard for notions of ownership (such as the Hillary Clinton campaign visual language, found images etc), cobbling together new combinations, reconfiguring and remixing, to explore new semantic vectors of attack.

- The swarm is operating in the adhocracy conditions identified by Bolman and Deal [36], where a flourishing of creativity and new directions is made possible by many nodes operating outside of a centralised, coordinated OODA loop. Combined with the speed of digital image production, simultaneous explorations of many information and semiotic messaging attack vectors is happening in a fully scalable capacity.

- Digital images are powerful objects of memetic warfare because they are inexpensive to both produce and remix, require little bandwidth to distribute, and are accessible on ubiquitous mobile media consumption platforms.

Existing work around internet memes is built on the dynamic of continual remix through viral, "spreadable" media (Jenkins, et $a l, 2013)$. Memetic warfare campaigns see this dynamic weaponised and undertaken with a design approach, where content is created with a particular semantic attack vector in mind. The occurrence of fast feedback loops and rapid iteration visible in \#DraftOurDaughters can be observed in content documented by both Rodley and Wiggins, where content around each situation documented is typically constructed with remixed media. In both case studies, digital images appear to be treated by users as essentially open source, with documented examples of content generated through remixing media from popular culture, news sources or other user-generated content, with little regard for notions of content ownership. This leads to content with powerful semiotic messaging attack vectors, as well-known images from popular culture are remixed with targeted political messaging, and inserted into public conversations.

\section{Anonymous}

Anonymity is a fundamental component of a successful memetic warfare campaign because it allows rapid scaling up of the swarm, as well as fast feedback loops, therefore building on the topologic and processual dynamics. As we observed in the \#DraftOurDaughters case study, the default state of 4chan as an anonymous platform re-enforces the distributed topologic dynamic through preventing centralisation mechanisms from appearing. Processually, anonymity creates a content production environment facilitating experimentation and fast feedback loops:

- The absence of identity leads to the disappearance of the entire social capital risk-reward economy. Anonymity operates as a flattener of the network, with each piece of content valued as is because there is no attachment to an account with a history able to generate social capital. The only kind of capital demonstrated is cultural capital through the use of subculture-specific communication tropes known to the community (Nissembaum and Shifman, 2017). For example, on /pol/ the swarm is unable to recognize any sort of social capital due to the absence of identity, but it recognizes the absence of cultural capital through the inability of a user to understand forum-specific communication tropes (such users are usually told to "lurk more").

- The mechanics of thread deletion mean that each new thread in the "/st/ stronger together" series essentially reboots conversation around the campaign, and all users participating receive new user IDs, wiping any social capital that may have been generated through content contribution in a specific thread.

- Anonymity blurs lines between combatants and civilians in the information war, exemplifying conditions of fourth generation warfare (Lind and Thiele, 2015). We do not know who started or contributed to \#DraftOurDaughters - it could have been anyone, Donald Trump's campaign team, an advertising agency, random groups of people, lone wolf media producers or all of the above. This demonstrates the importance of John Robb's observation of the "plausible promise" dynamic in combination with the mechanism for effortless network scaling as a key feature to the swarm.

Swarms around memetic warfare campaigns typically include users with obfuscated identity. In Rodley's study, content on both sides of the Israel-Gaza conflict was contributed by a disparate patchwork of users, some identifiable as state and non-state groups, and some using anonymous Twitter accounts. This does not account for possible false identity accounts used to deploy content or generate false community sentiment, as seen in \#DraftOurDaughters. Similarly, content documented in Wiggins' study has no indicator of authorship, meaning content could be created by any state or non-state entity. Absent in both studies is observable hierarchy or centralized decision-making in content production. The closest 
dynamic is found in Wiggins' study, where the content observed is from a Twitter account aggregating content from Russian social media and deploying on Twitter. Anonymity is also a key dynamic to Massanari's (2017) documentation of swarming on Reddit, where users operate anonymously for the most part, as user accounts are typically pseudonyms [37].

\section{Decision-making through memetics}

As a component, memetic decision-making presupposes an OODA loop built entirely on copying successful tactics rather than a rigid operational doctrine. In other words, the observe and orient feedback loop stages of a network engaged in a memetic warfare campaign have to involve the copying and iteration of successful attack vectors. In a processual sense, 4chan has an established and accepted culture of users copying content formats, while balancing this with constant tinkering and variation to find new vectors of resonance (Milner, 2013c). Normally, framing a stable memetic message in an environment open to multiple networks is costly because of the constant frame overflows, such as dilution of intended meaning, generated by the heterogeneity of the actors involved (Callon, 1998). Crucially, memetic swarms deal with frame overflows by rapidly circulating feedback across the surface of the swarm, in effect turning the weakness into an advantage. Memetic warfare leverages this culture and sensibility to generate a media campaign appearing highly coordinated despite being driven by the distributed topology of the chaotically organised underlying swarm of users:

- Copying rather than coordinated decision-making drives all facets of the campaign, from production of content to strategies of deployment to wider audiences, particularly on Twitter.

- Thread deletion and creation of sequel threads tests the adoption of content, as content must regain momentum at the beginning of each new thread. Only the most well adopted content messaging vectors survive creation of a new thread.

- The end point of a campaign is found when the memetic intensity of a swarm begins to subside as sequel threads fail to regain the momentum of previous threads.

- 4chan's culture of trolling (Phillips, 2013; Milner, 2013b; Massanari, 2017) means that even discussion approving or dismissing possible directions cannot be taken at face value. Actions copied multiple times are the only indicator of adoption.

- True consensus decision-making is indicated through the stabilization of content. In the case of \#DraftOurDaughters, early campaign material was varied in terms of visual style, language and concept, but as successful angles were discovered and copied, the consistency evolved into a template.

- In turn, stabilization is viewed by the 4chan community as a property to be exploited. This is typical of Arquilla and Ronfeldt's recognizing swarms as particularly strong in disrupting coordinated and well-resourced stable systems. In the case of \#DraftOurDaughters, the swarm successfully uses the Hillary Clinton campaign visual language (a visual signifier of coordination and resource) as a weakness.

In any given ongoing memetic warfare campaign, particular semantic tropes emerge and become memetic as the swarm copies what it finds to be a successful semantic attack vector. In Wiggins' content, use of images of Russian president Vladimir Putin become widespread as the swarms, either favoring Ukraine or favoring Russia, gravitate towards using him as a message container and figure to remix with popular culture [38]. Rodley's study finds that memetic content around the Israel-Gaza conflict developed as the swarms adopted remix of popular culture images as a key tactic of resonance generation, with infographics also emerging as content format frequently deployed. While \#DraftOurDaughters differs from these studies in the sense that content is produced by only one side of the conflict, content generation by the swarm through copying the actions of others rather than coordinated, doctrine-driven consensus decision-making is the key driver of content development and refinement.

\section{Conclusion}

We have proposed a coherent and systemic perspective on the logistics of a memetic warfare campaign by developing a framework of integrated topologic and processual dynamics. Public operations, open source protocol, anonymous participants and decision-making through memetics are evidenced as key to conducting a successful memetic warfare campaign. The case study of \#DraftOurDaughters exemplifies this framework, which maps to other documented case studies of memetic warfare. 4chan in particular is identified as an environment fostering these key dynamics, with platforms such as Reddit and Twitter also evidenced as platforms with somewhat similar qualities. Also identified as key to memetic warfare is the weaponization of the natural language of the Internet, harnessing continual copying and remixing of content and using a design process to create semantically targeted content. This suggests memetic warfare as a critical topic of interest under the larger umbrella of information operations. FM

\section{About the authors}

Travis Wall is a Ph.D. candidate in the school of The Arts, English and Media at the University of Wollongong. He is a graphic designer interested in the trajectory of visual communication and design process in a context of digital media. His research looks at the manifestation of design process in online communities and user generated media, particularly in information warfare. He also experiments with creation of online digital artefacts to understand design and development process in this space.

E-mail: twall [at] uow [dot] edu [dot] au

Dr. Teodor Mitew is a Senior Lecturer in digital media in the School of the Arts, English and Media at the University of Wollongong. His research background is in actor network theory and internet studies. His current projects range across the Internet of things, swarm content networks, memetic warfare, object oriented ontology, and smart textiles.

E-mail: tmitew [at] uow [dot] edu [dot] au

\section{Notes}


1. Giesea, 2015, p. 70.

2. Ibid.

3. Baran, 1962, pp. 3-4.

4. Baran, 1962, p. 3.

5. Ibid.

6. Baran, 1962, pp. 18-22.

7. Castells, 2004, p. 3.

8. Bolman and Deal 2008, pp. 78-86.

9. Raymond, 2001, p. 2.

10. Ibid.

11. Bolman and Deal, 2008, p. 86 .

12. Weber, 2004, p. 64.

13. Robb, 2007, p. 74.

14. Robb, 2007, pp. 74-75.

15. Robb, 2007, p. 116.

16. Robb, 2007, pp. 94-110.

17. Arquilla and Ronfeldt, 2000, pp. 1-3.

18. Arquilla and Ronfeldt, 2000 , p. 3.

19. Rheingold, 2002, pp. 157-164.

20. Arquilla and Ronfeldt, 2000, p. 27.

21. Arquilla and Ronfeldt, 2000, pp. 21-23.

22. Arquilla and Ronfeldt, 2000, pp. 45-46.

23. Flaherty, 2010, p. 5.

24. Bolman and Deal, 2008, p. 86.

25. Robb, 2007, pp. 118-119.

26.Bolman and Deal, 2008, p. 86.

27. Raymond, 2001, p. 9.

28. Dubberly, 2008, p. 46.

29. Lawson, 2005, p. 34.

30. Lawson, 2005, p. 37.

31. Lawson, 2005, pp. 36-38.

32. Lawson, 2005, p. 48.

33. Hine, et al., 2017, pp. 6-7.

34. Hine, et al., 2017, p. 5.

35. Wiggins, 2016, p. 5.

36. Bolman and Deal, 2008, p. 86.

37. Massanari, 2017, p. 335.

38. Wiggins, 2016, pp.19-20.

\section{References}

John Arquilla and David Ronfeldt, 2000. Swarming and the future of conflict. Santa Monica, Calif.: RAND Corporation, at https://www.rand.org/content/dam/rand/pubs/documented briefings/2005/RAND DB311.pdf, accessed 4 December 2017.

Paul Baran, 1962. "On distributed communications networks" Santa Monica, Calif.: RAND Corporation, at http://www.rand.org/content/dam/rand/pubs/papers/2005/P2626.pdf, accessed 4 December 2017.

Lee Bolman and Terrence Deal, 2008. Reframing organizations: Artistry, choice, and leadership. Fourth edition. San Francisco, Calif.: Josey-Bass. 
John Boyd, 1986. "Patterns of conflict," presentation, unpublished collection of diagrams, dated December 1986.

Axel Bruns, 2010. "Distributed creativity: Filesharing and produsage," In: Stefan Sonvilla-Weiss (editor). Mashup cultures. Wein: Springer-Verlag, pp. 24-37.

doi: https://doi.org/10.1007/978-3-7091-0096-7 2, accessed 5 April 2018.

Michel Callon, 1998. "An essay on framing and overflowing: Economic externalities revisited by sociology," Sociological Review, volume 51, number S1, pp. 244-269.

doi: https://doi.org/10.1111/j.1467-954X.1998.tb03477.x, accessed 5 April 2018

Michel Callon and John Law, 2004. "Introduction: Absence - presence, circulation, and encountering in complex space," Environment and Planning D: Society and Space, volume 22, number 1, pp. 3-11. doi: https://doi.org/10.1068/d313, accessed 5 April 2018.

Manuel Castells, 2004. "Informationalism, networks, and the network society: A theoretical blueprint," In: Manuel Castells (editor), The network society: A cross-cultural perspective. Cheltenham: Edward Elgar, pp. 3-48. doi: https://doi.org/10.4337/9781845421663.00010, accessed 5 April 2018.

Hugh Dubberly, 2008. "How do you design? A compendium of models," at http://www.dubberly.com/wpcontent/uploads/2008/06/ddo designprocess.pdf, accessed 4 December 2017.

Chris Flaherty, 2010. "Command, influence and information in 3D tactics," Journal of Information Warfare, volume 9, number 1, at https://www.jinfowar.com/journal/volume-9-issue-1/command-influence-information-3d-tactics, accessed 4 December 2017.

Noam Gal, Limor Shifman and Zohar Kampf, 2015. "'It gets better': Internet memes and the construction of collective identity," New Media \& Society, volume 18, number 8, pp. 1,698-1,714. doi: https://doi.org/10.1177/1461444814568784, accessed 5 April 2018.

Jeff Giesea, 2015. "It's time to embrace memetic warfare," Defence Strategic Communications, volume 1, number 1, pp. 67-75; version at https://www.stratcomcoe.org/jeff-giesea-its-time-embrace-memetic-warfare, accessed 5 April 2018.

Tim Hains, 2016, "Jill Stein: Trump is less dangerous than Clinton; She will start nuclear war with Russia," RealClearPolitics (12 October), at https://www.realclearpolitics.com/video/2016/10/12/jill stein hillary clintons declared syria policy could start a nuclear war.html, accessed 4 December 2017

Jana Herwig, 2011, "The archive as the repertoire: Mediated and embodied practice on Imageboard 4chan.org," In: Günther Friesinger, Johannes Grenzfurthner and Thomas Ballhausen (editors). Mind and matter: Comparative approaches towards complexity. Bielefeld: transcript Verlag, pp. 39-56.

Gabriel Emile Hine, Jeremiah Onaolapo, Emiliano De Cristofaro, Nicolas Kourtellis, Ilias Leontiadis, Riginos Samaras, Gianluca Stringhini and Jeremy Blackburn, 2017. "Kek, cucks, and god emperor Trump: A measurement study of 4chan's politically incorrect forum and its effects on the Web," Proceedings of the Eleventh International AAAI Conference on Web and Social Media (ICWSM 2017), pp. 92-101, and at https://aaai.org/ocs/index.php/ICWSM/ICWSM17/paper/download/15670/14790, accessed 5 April 2018.

Henry Jenkins, 2006. Convergence culture: Where old and new media collide. New York: New York University Press.

Henry Jenkins, Sam Ford and Joshua Green, 2013. Spreadable media: Creating value and meaning in a networked culture. New York: New York University Press.

Kevin Kelly, 2008. "Better than free," Edge.org (5 February), at https://www.edge.org/conversation/kevin kelly-betterthan-free, accessed 4 December 2017.

Michele Knobel and Colin Lankshear, 2007. "Online memes, affinities, and cultural production," In: Michele Knobel and Colin Lankshear (editors). A new literacies sampler. New York: Peter Lang, pp. 199-227.

Lee Knuttila, 2011. "User unknown: 4chan, anonymity and contingency," First Monday, volume 16, number 10, http://firstmonday.org/article/view/3665/3055, accessed 5 April 2018. doi: http://dx.doi.org/10.5210/fm.v16i10.3665, accessed 5 April 2018.

Bryan Lawson, 2005. How designers think: Demystifying the design process. Fourth edition. Oxford: Architectural Press.

William S. Lind and Gregory A. Thiele, 2015. 4th generation warfare handbook. Kouvola, Finland: Castalia House.

Adrienne Massanari, 2017. "\#Gamergate and the fappening: How Reddit's algorithm, governance, and culture support toxic technocultures," New Media \& Society, volume 19, number 3, pp. 329-346. doi: https://doi.org/10.1177/1461444815608807, accessed 5 April 2018.

Ryan Milner, 2013a. "Pop polyvocality: Internet memes, public participation, and the Occupy Wall Street movement," International Journal of Communication, volume 7, pp. 2,357-2,390, and at http://ijoc.org/index.php/ijoc/article/view/1949, accessed 5 April 2018.

Ryan Milner, 2013b. "Hacking the social: Internet memes, identity antagonism, and the logic of lulz," Fibreculture Journal, number 22, http://twentytwo.fibreculturejournal.org/fcj-156-hacking-the-social-Internet-memes-identity-antagonism-andthe-logic-of-|ulz|, accessed 5 April 2018.

Ryan Milner, 2013c. "Media lingua franca: Fixity, novelty, and vernacular creativity in Internet memes," Selected Papers of Internet Research, at http://spir.aoir.org/index.php/spir/article/view/806/390, accessed 5 April 2018.

Asaf Nissenbaum and Limor Shifman, 2017. "Internet memes as contested cultural capital: The case of 4chan /b/ board," New Media \& Society, volume 19, issue 4, pp. 483-501.

doi: https://doi.org/10.1177/1461444815609313, accessed 5 April 2018.

Eric Raymond, 2001. The cathedral and the bazaar: Musings on Linux and Open Source by an accidental revolutionary. Revised edition. Beijing: O'Reilly. 
Howard Rheingold, 2002. Smart mobs: The next social revolution. Cambridge, Mass.: Perseus.

John Robb, 2016, "Welcome John Robb (/u/John_Robb), author and entrepreneur. Ask Him Anything!" Reddit (12 August), at https://www.reddit.com/r/The Donald/comments/4xgeb5/welcome_john_robb ujohn_robb author and/, accessed 5 April 2018.

John Robb, 2007. Brave New War: The next stage of terrorism and the end of globalization. Hoboken, N.J..: Wiley.

Chris Rodley, 2016. "When memes go to war: Viral propaganda in the 2014 Gaza-Israel conflict," Fibreculture Journal, number 27, http://twentyseven.fibreculturejournal.org/2016/03/18/fcj-200-when-memes-go-to-war-viral-propaganda-inthe-2014-gaza-israel-conflict/, accessed 5 April 2018.

U.S. Congress, 2016. "All bill (except text) for H.R.4478 - Draft America's Daughters Act of 2016," at https://www.congress.gov/bill/114th-congress/house-bill/4478/all-info, accessed 5 April 2018.

Dan Ward, 2011. "Fast, inexpensive, simple and tiny, FIST at 5: Looking back, looking ahead," Defense AT\&L, volume 40, number 3, pp. 33-37.

Steven Weber, 2004. The success of open source. Cambridge, Mass.: Harvard University Press.

Bradley Wiggins, 2016. "Crimea River: Directionality in memes from the Russia-Ukraine conflict," International Journal of Communication, volume 10, pp-451-485, and at http://ijoc.org/index.php/ijoc/article/view/4103, accessed 4 December 2017.

Peter Lamborn Wilson,1997. "A network of castles," at http://www.ljudmila.org/nettime/zkp4/17.htm, accessed 4 December 2017.

\section{Appendix}

Concept exploration and refinement

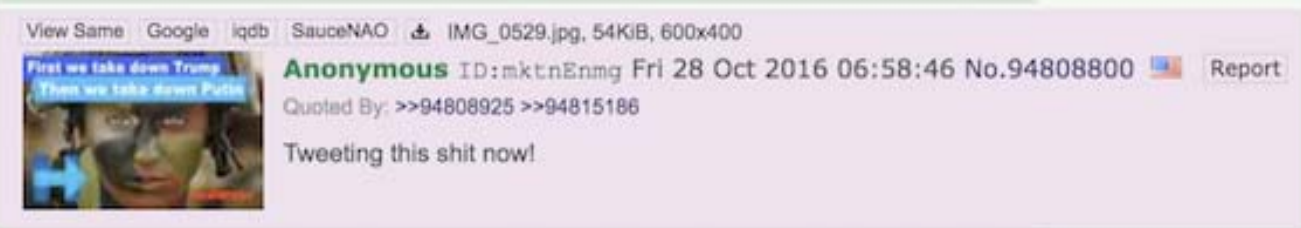




\section{@OnMessageForHer \#FightForHer \#EnlistForHer} \#ImWithHer \#HillaryClinton Why I'd vote Hillary if only I were American!

\section{First we take down Trump}
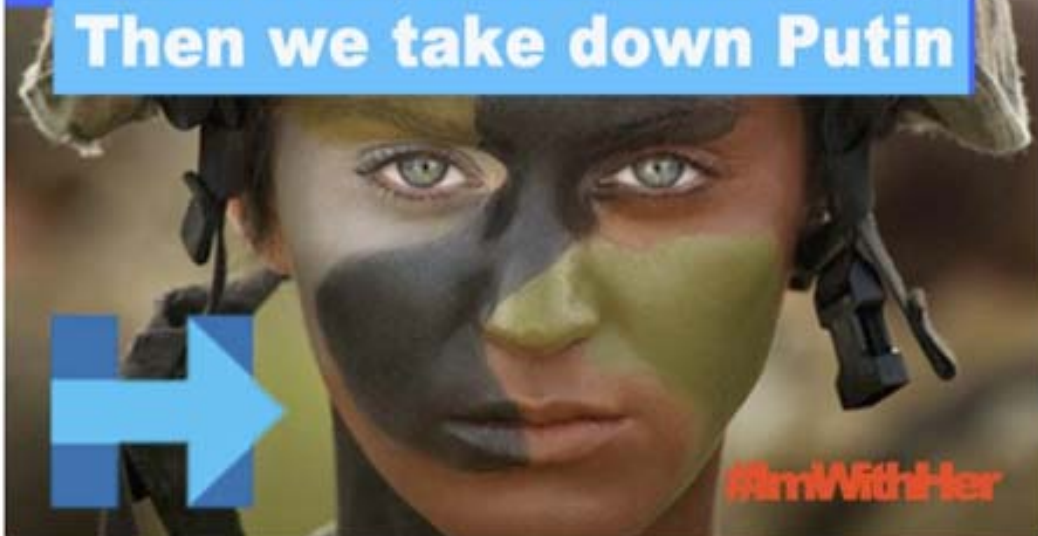

LACE

1

$12: 47$ PM - 27 Oet 2016

\& $\quad$ เ?

Figure 7: A /pol/ user prototyping content and deploying to Twitter. Post archived at http://archive.4plebs.org/pol/thread/94805438/\#94808800. Tweet archived at http://archive.is/q23f9.

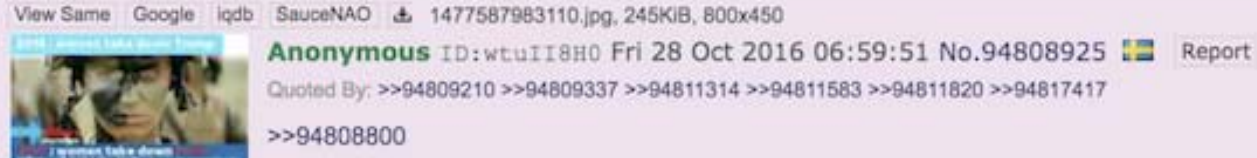

Figure 8: Iteration of Figure 7 image with altered slogans. Post archived at http://archive.4plebs.org/pol/thread/94805438/\#94808925.

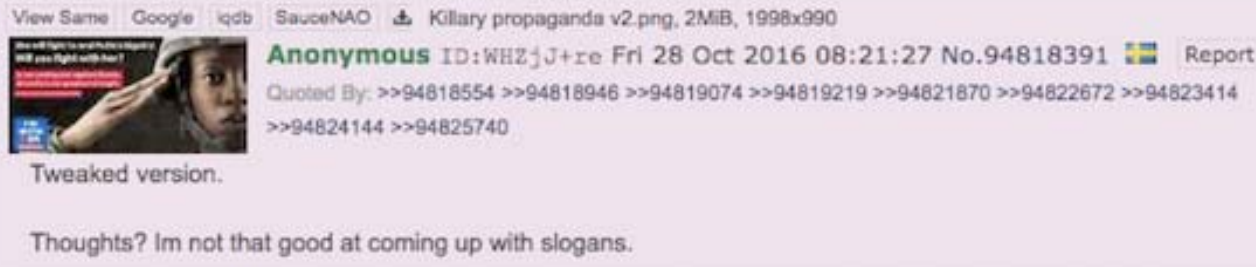
Cuoted By, >>94818554 >>94818946 >>94819074 >>948192.19 >>94821870 >>94822672 >>94823414 $\gg 994824144>>94825740$

Tweaked version

Thoughts? Im not that good at coming up with slogans. 
Our armed forces need more women \#FightForHer
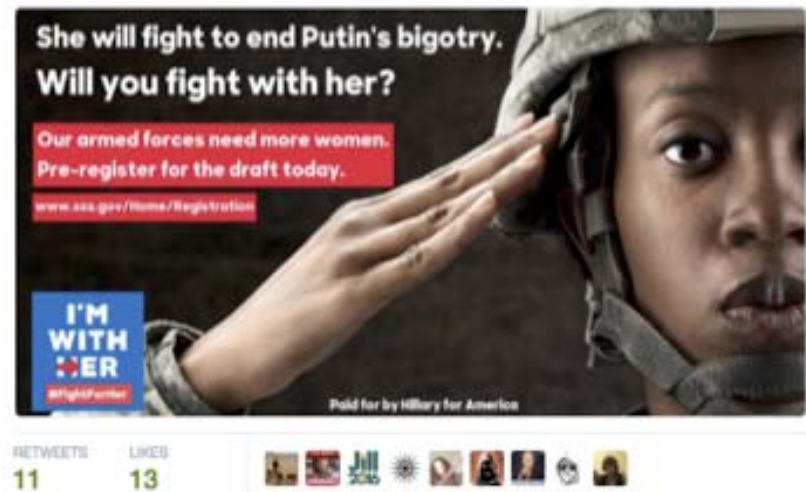

2:16 PM-27 Oct 2016

(4) 2711

Q $13 \quad \ldots$

Figure 9: A /pol/ user prototyping content and deploying to Twitter. Post archived at http://archive.4plebs.org/pol/thread/94816222/\#94818391. Tweet archived at http://archive.is/nr5Sz.

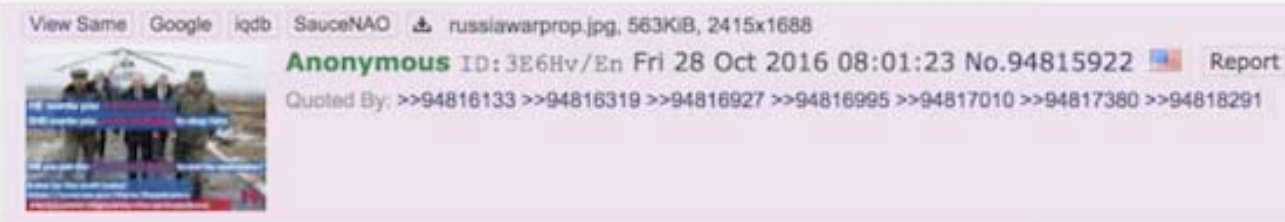

Rodger Cuckman

\#FightForHer \#EnlistForHer \#NoFlyZone

\#womensrights

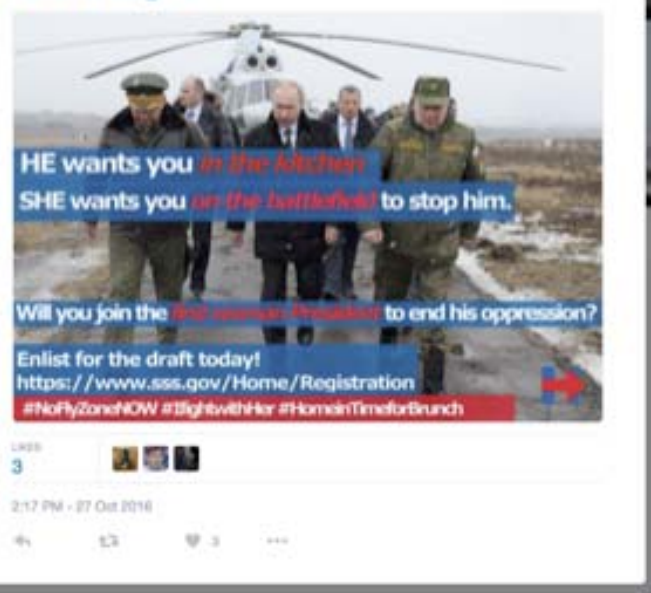

Figure 10: A /pol/ user prototyping content and deploying to Twitter. Post archived at http://archive.4plebs.org/pol/thread/94805438/\#94815922. Tweet archived at http://archive.is/pxpU. 


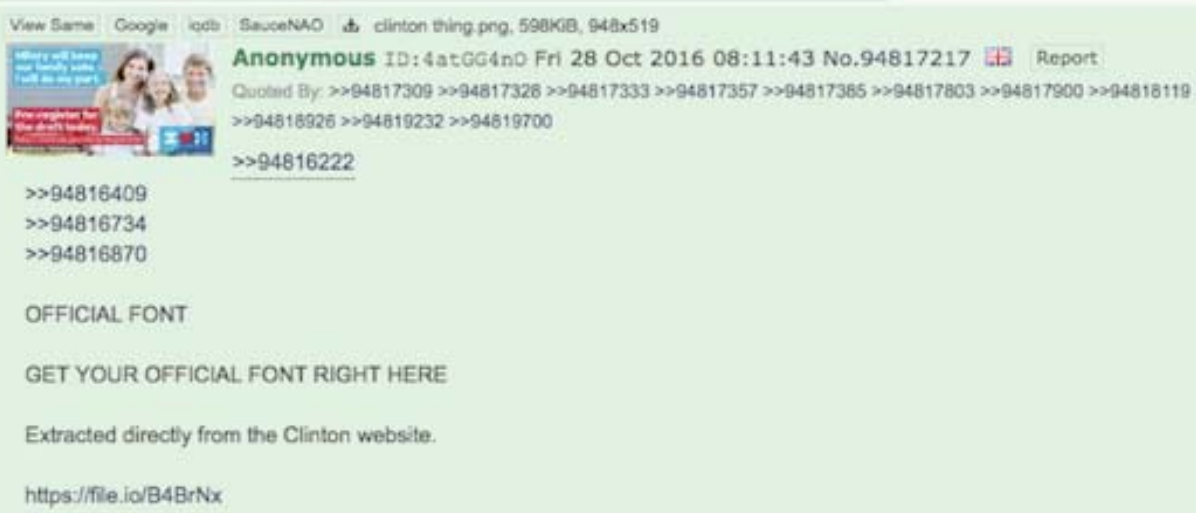

Figure 11: The appearance of the Hillary Clinton campaign font posted for download. Post archived at http://archive.4plebs.org/pol/thread/94816222/\#94817217.

\section{Campaign concept development}

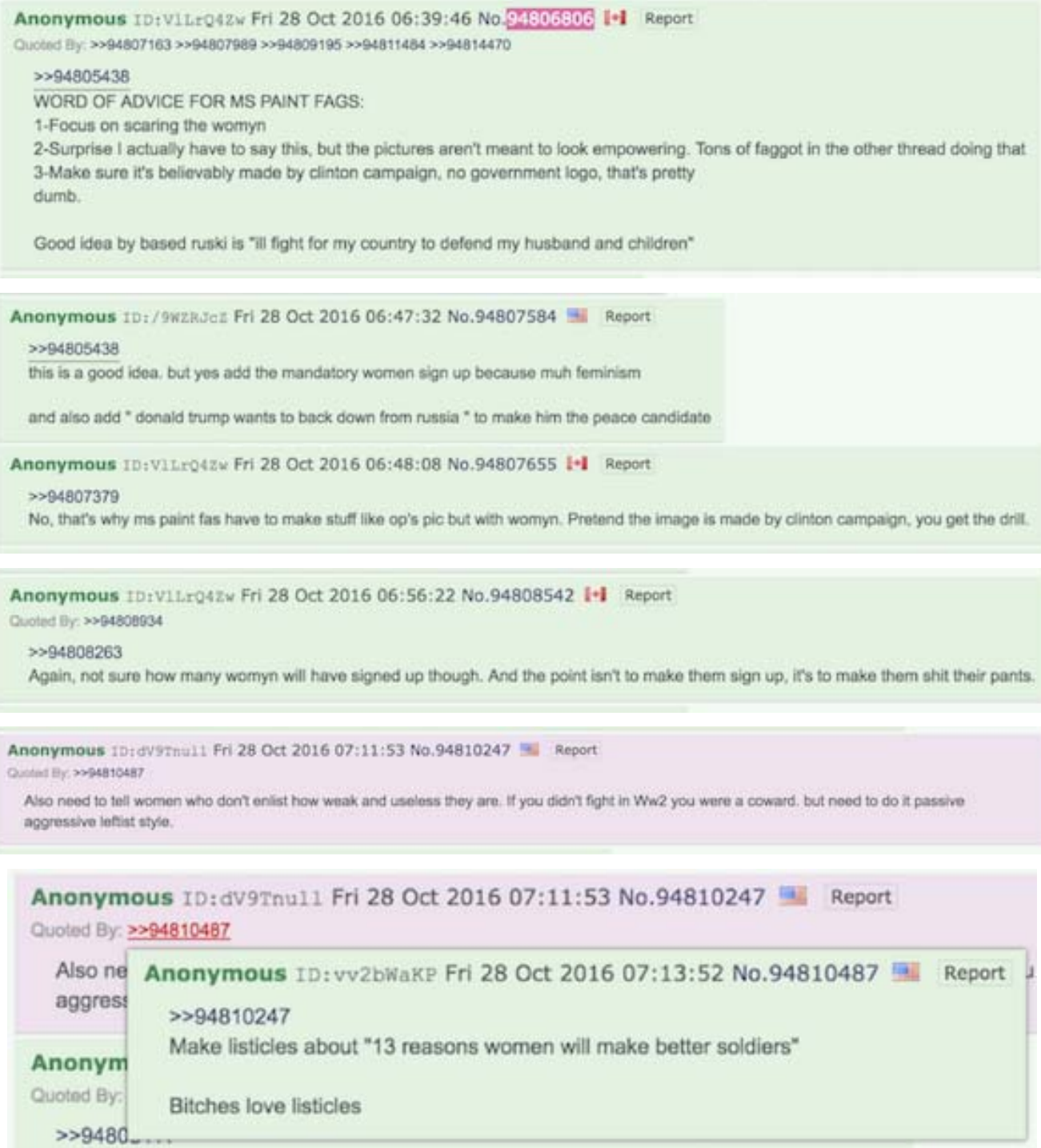

Figure 12: Various posts from the original threads creating and developing concepts. 


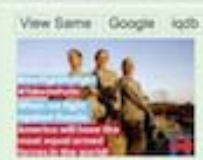

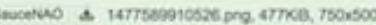

Anonymous rorwturisio Fri 28 Oct 2016 06:56:57 No.94808611 :Z Report

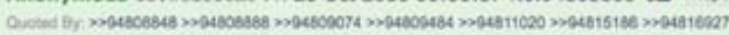

$>>94807989$

Family is too while and the fonts are wrong.

Official forth: httpsiliny moxtape moeligivzs zip

Try to spin the "war efforr to be against putins "bigotry" rather than for safety Make the liberais question how mucth they are wiling to sacrifce to defend degeneracy

Pic related is pood.

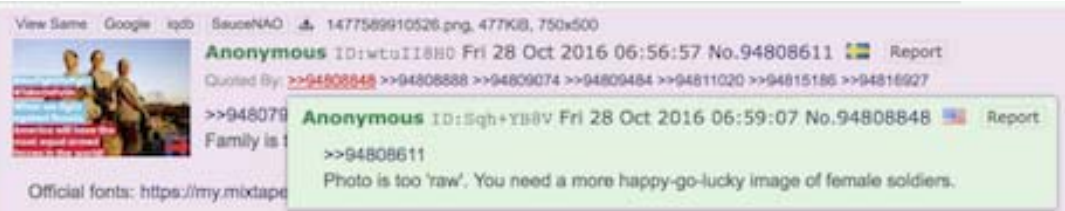

Try to spin the "war effort to be against putins "bigoty' rather than for safety. Make the lberals question how much they are willing to sacrifice to defend degeneracy.

Ple related is good

\begin{tabular}{|c|c|c|}
\hline & 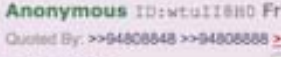 & 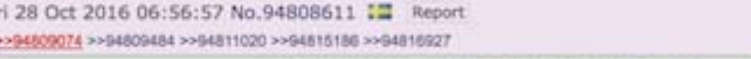 \\
\hline 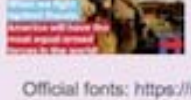 & $\begin{array}{l}>>94807989 \\
\text { Family is too white and the fonts }\end{array}$ & $\begin{array}{l}\text { Anonymous } 10+386 \mathrm{Bv} / \mathrm{En} \text { Fri } 28 \text { Oct } 2016 \text { 07:01:14 No.94809074 al Report } \\
\text { >P94808611 } \\
\text { this seams almost too snarky }\end{array}$ \\
\hline
\end{tabular}

Try to spin the "War effort to be against putins "bigotry" rather than for safety Make the liberals question how much they are wiling to sacrifce to defend degeneracy

Pic related is good.

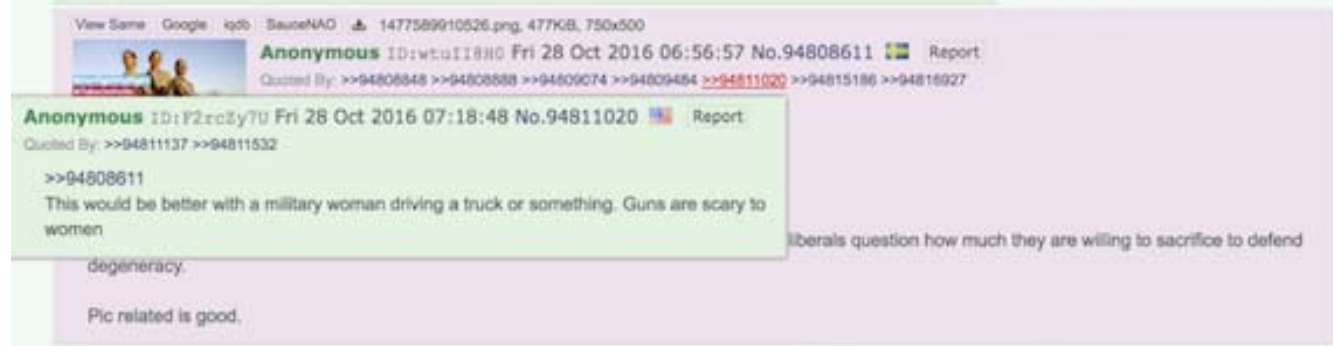

Figure 14: Content posted to /pol/, followed by critique and development. Post archived at http://archive.4plebs.org/pol/thread/94805438/\#94808611.

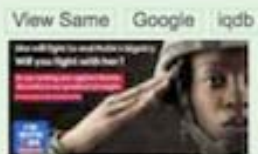

Tweaked version.

Thoughts? Im not that good at coming up with slogans.

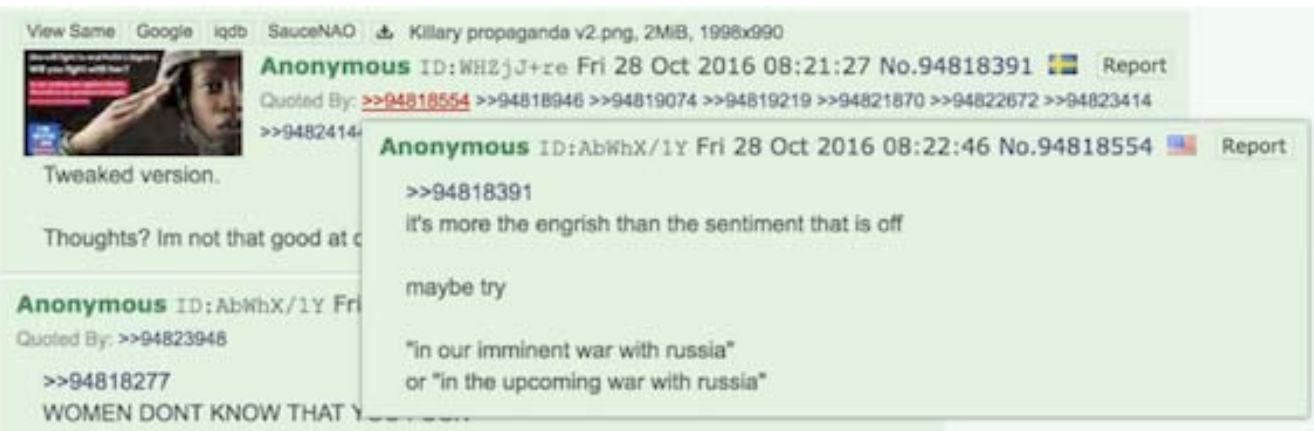




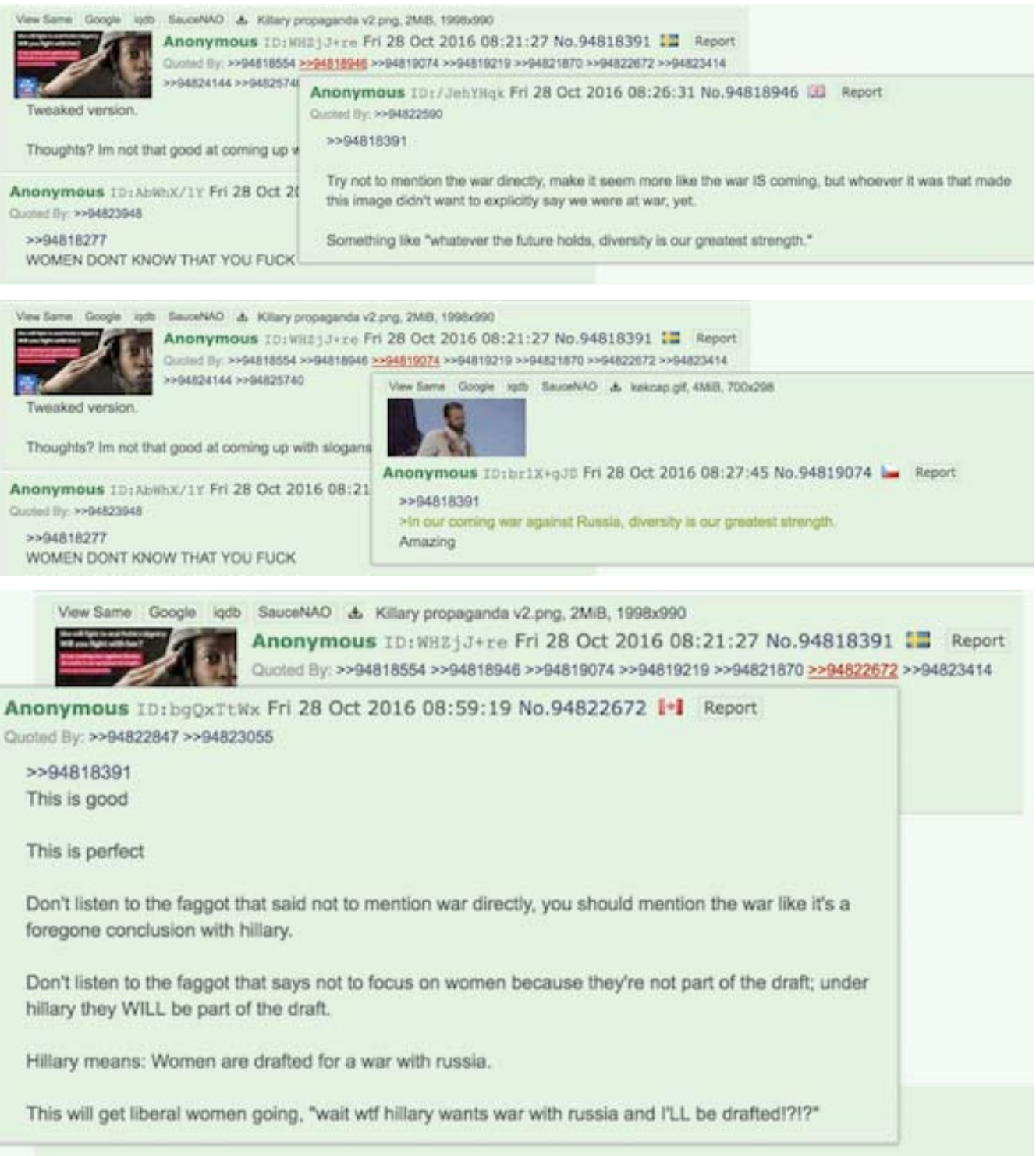

Figure 15: Content posted to /pol/, followed by critique and development. Post archived at http://archive.4plebs.org/pol/thread/94816222/\#94818391.

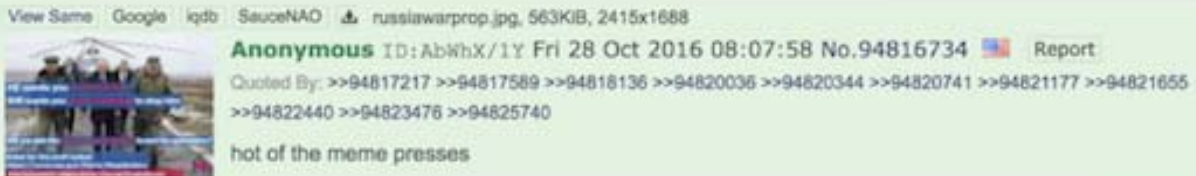




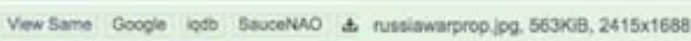

Anonymous ID:AbWhX/1Y Fri 28 Oct 2016 08:07:58 No.94816734 4 th Report

extiven:

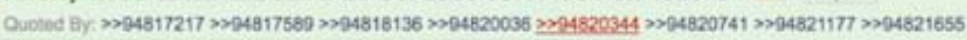

Anonymous ID:EnVYar6y Fri 28 Oct 2016 08:39:48 No.94820344 = Report

Quched By, $>>94820650>>04821038$

$>994820036$

$>94816734$

It appeals a lot to wornen I think its good..

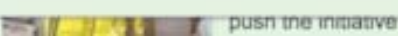

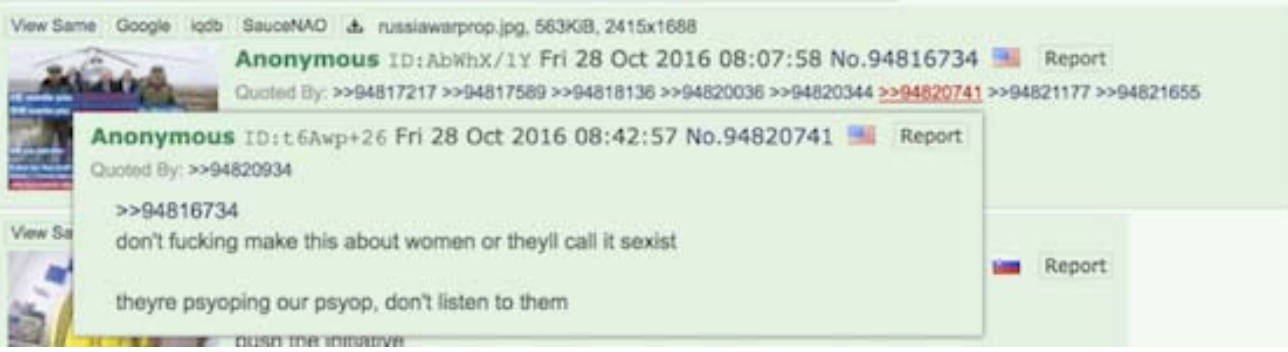

Figure 16: Content posted to /pol/, followed by critique and development. Original posts and feedback archived at http://archive.4plebs.org/pol/thread/94816222/\#94816734.

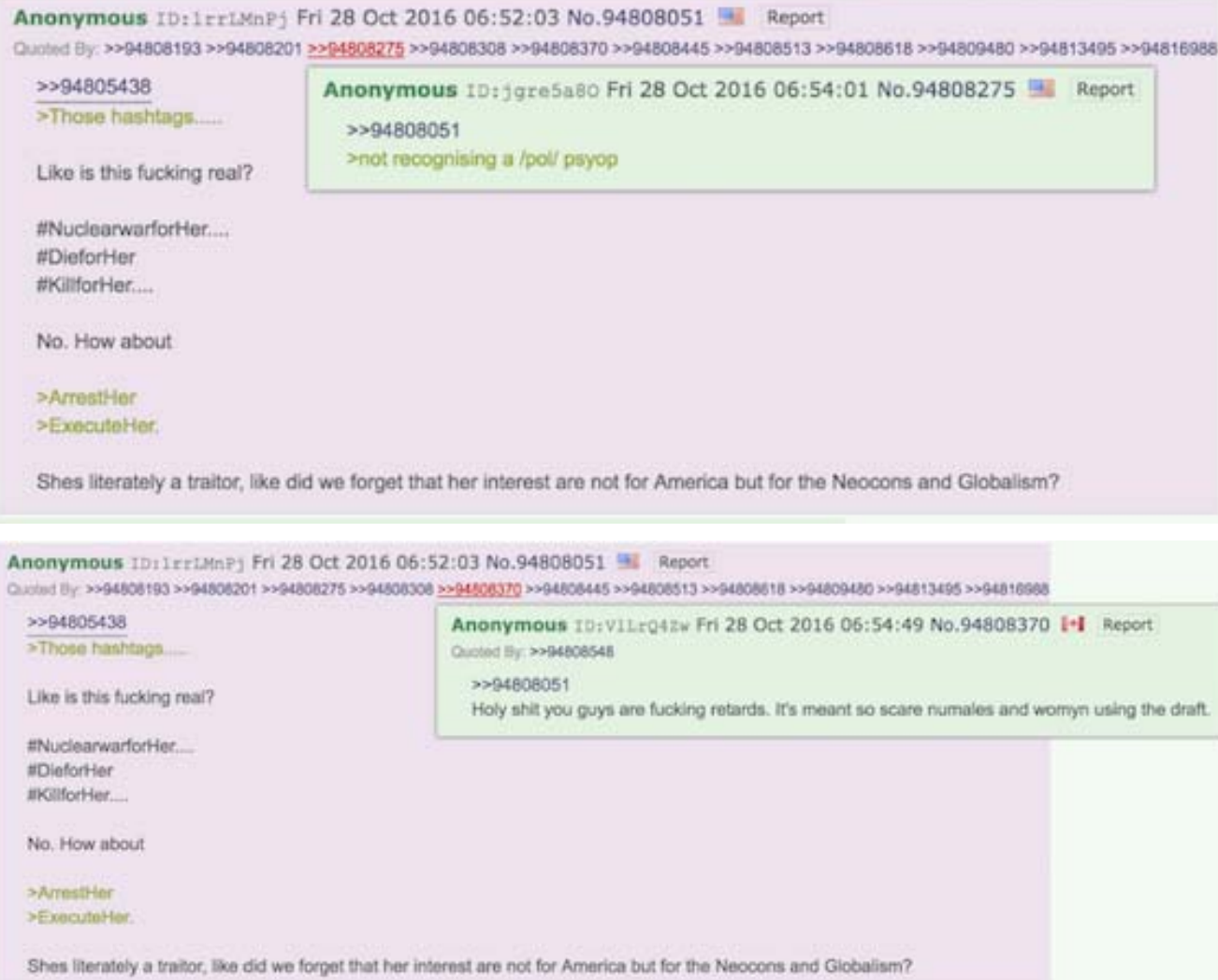




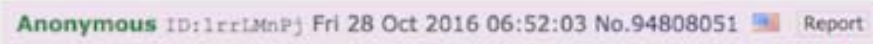

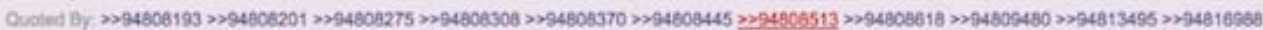

Anonymous rD:bRiz8t4D Fri 28 Oct 2016 06:56:06 No.94808513 19 Report

$>>94808051$

are you autistic? not understanding social cues is a sign of autism anon.

"NuclearwarforHer.

\#Diafortier

wKillforHer...

No. How about

$>$ Arresther

$>$ ExecuteHer

Shes literately a trattor, like did we forget that her interest are not for America but for the Neocons and Globalism?

Figure 17: Negative feedback in thread. Post archived at http://archive.4plebs.org/pol/thread/94805438/\#94808051.

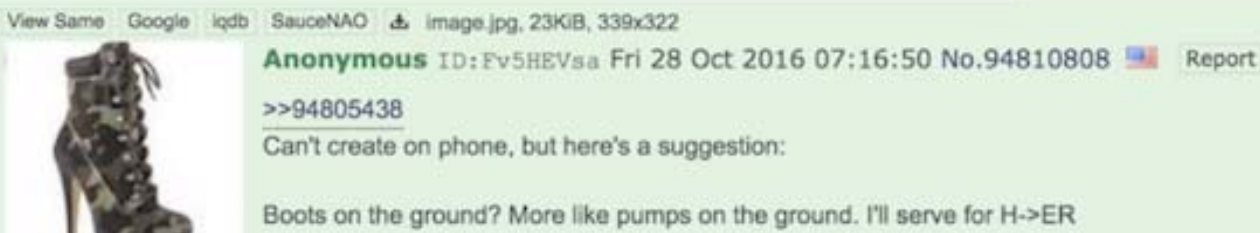

Figure 18: Ideas proposed but not copied or followed through. Post archived at http://archive.4plebs.org/pol/thread/94805438/\#94810808.

\section{Emergence of \#DraftOurDaughters hashtag}

The initial posting of the \#DraftOurDaughters hashtag followed by a string of comments. Post archived at http://archive.4plebs.org/pol/thread/94816222/\#94818458

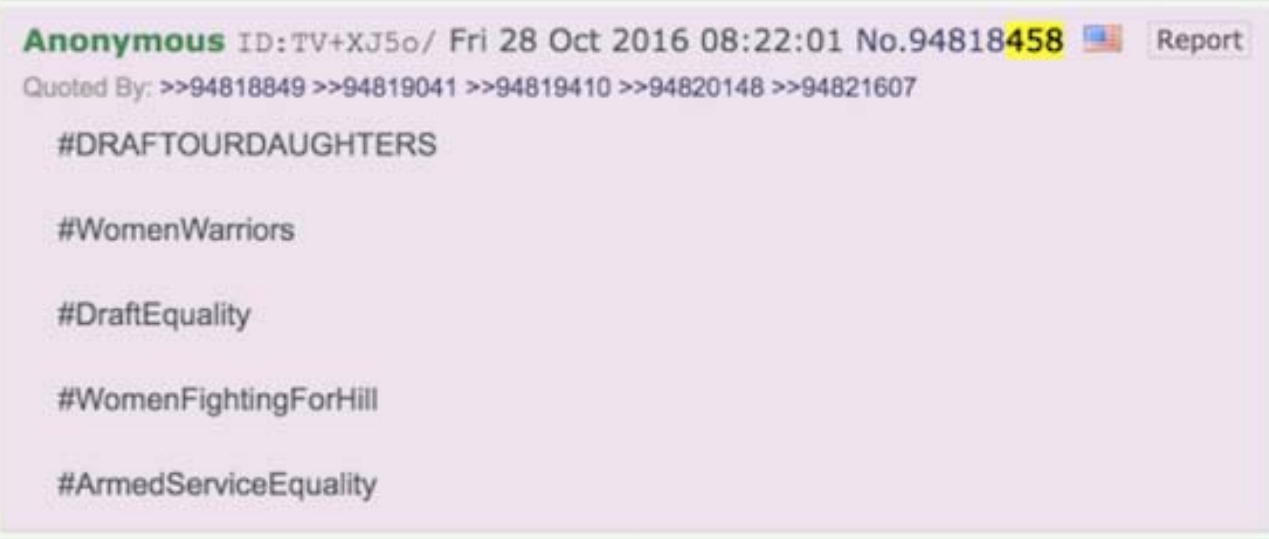




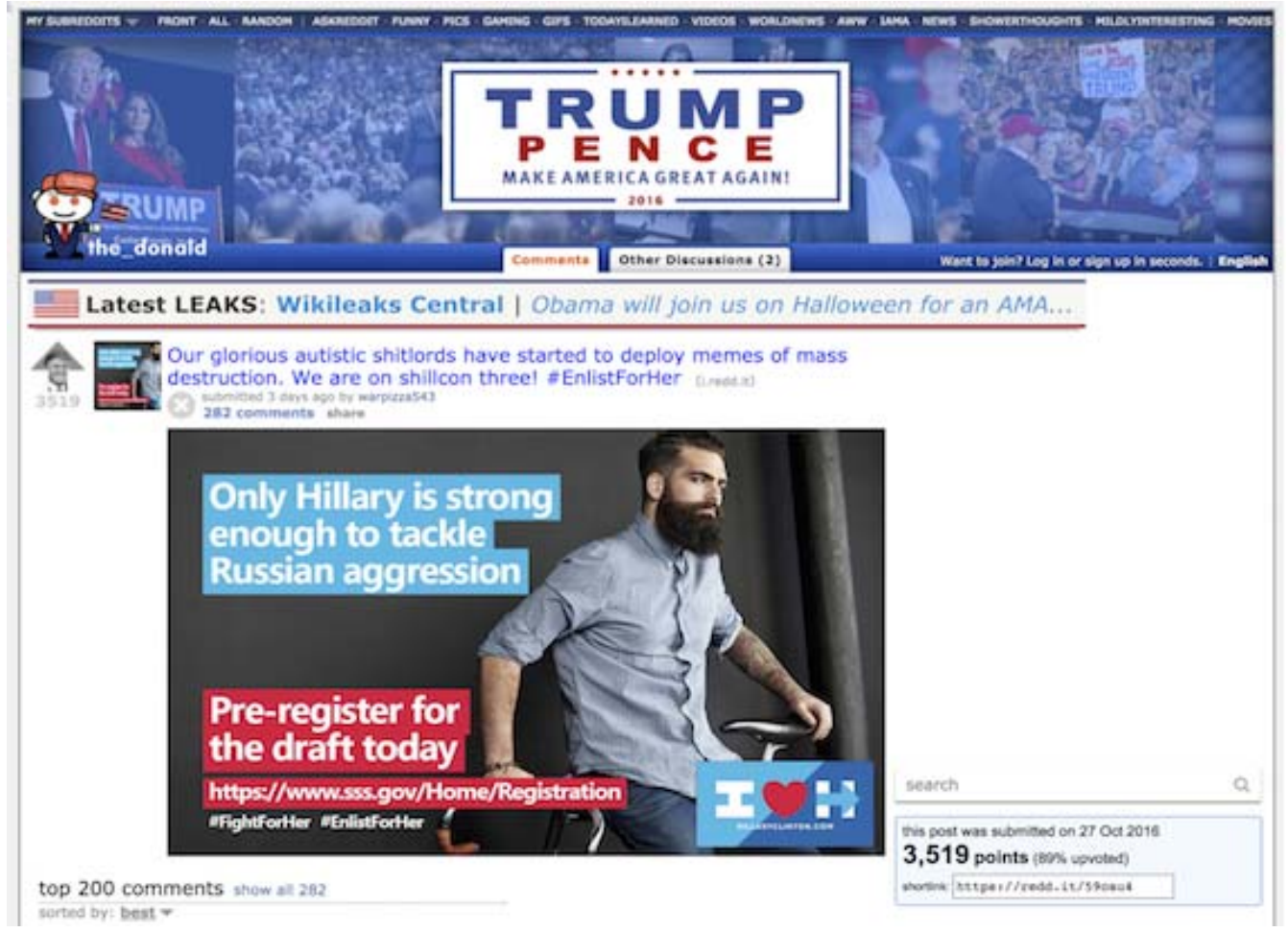

Figure 21: Thread on Reddit similar to threads on 4chan. Page archived at http://archive.is/ZLXff.

\section{Organising and producing memes}

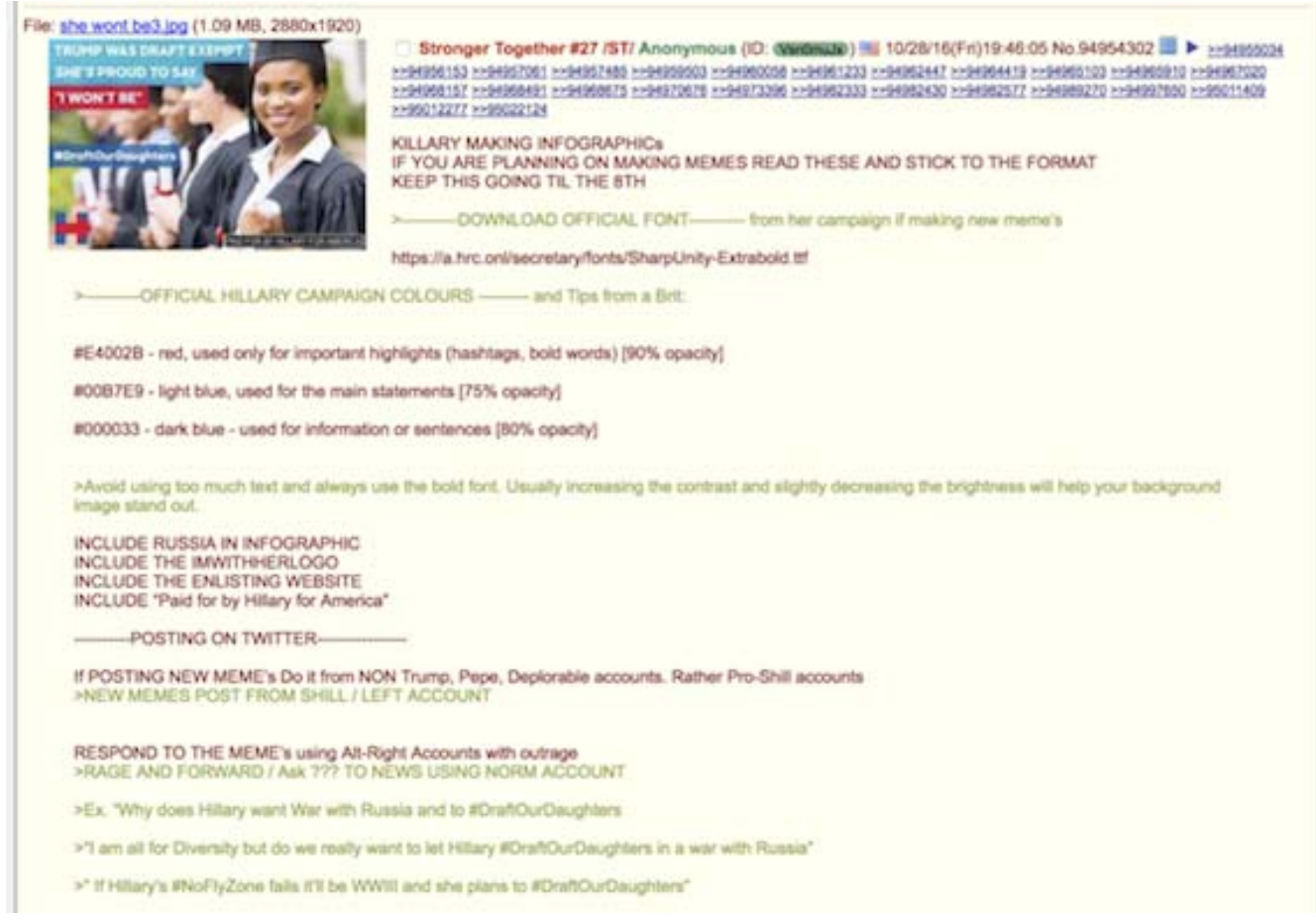

Figure 22: Thread on Reddit similar to threads on 4chan. Page archived at http://archive.is/gMi8g. 


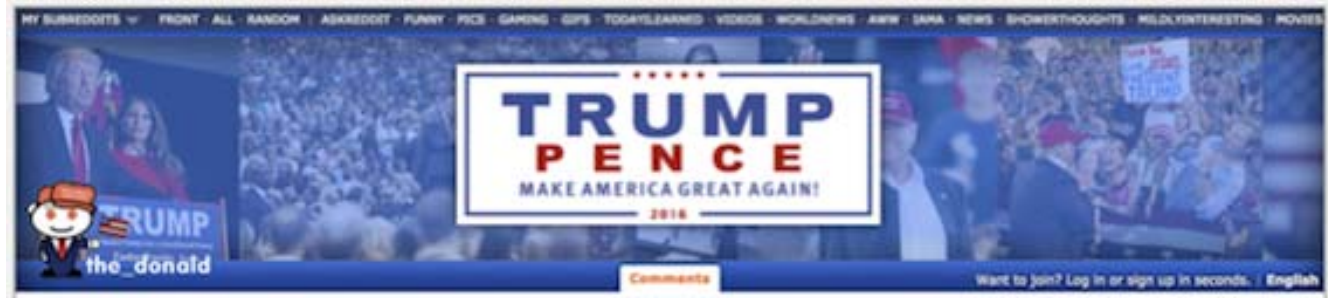

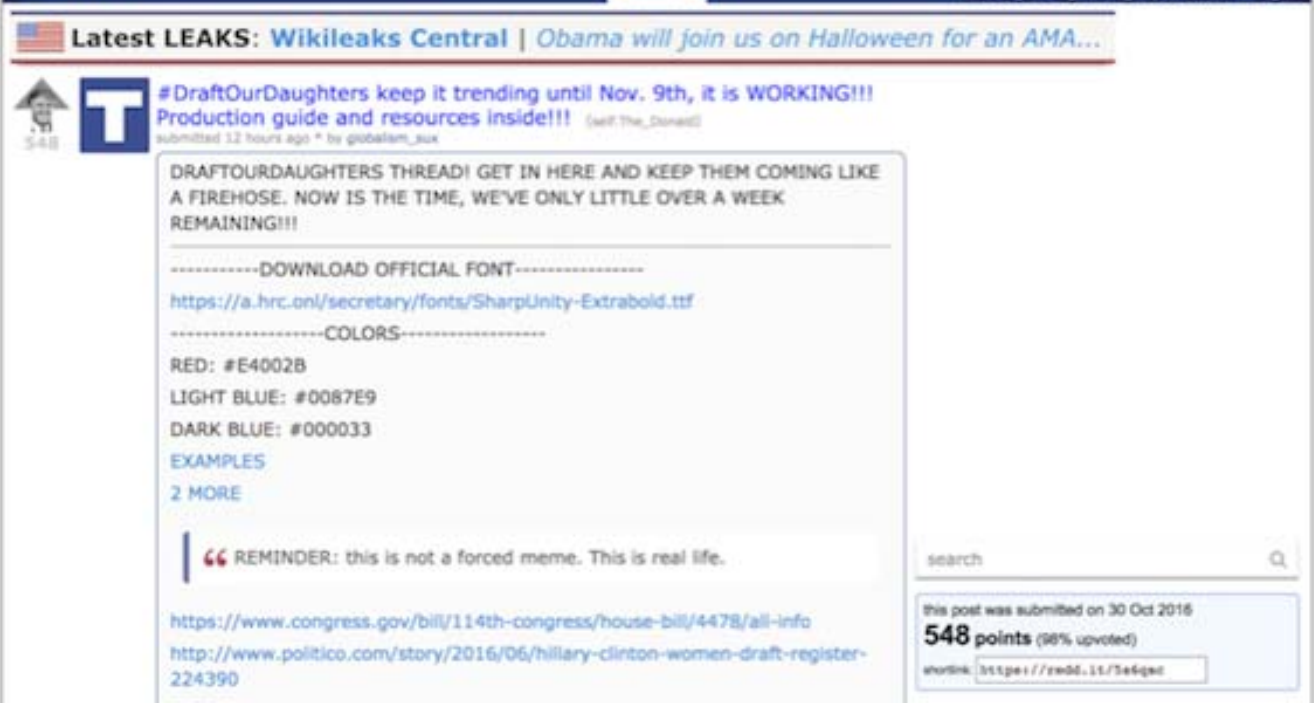

Figure 23: Thread on Reddit similar to threads on 4chan. Page archived at http://archive.is/GIK3z.

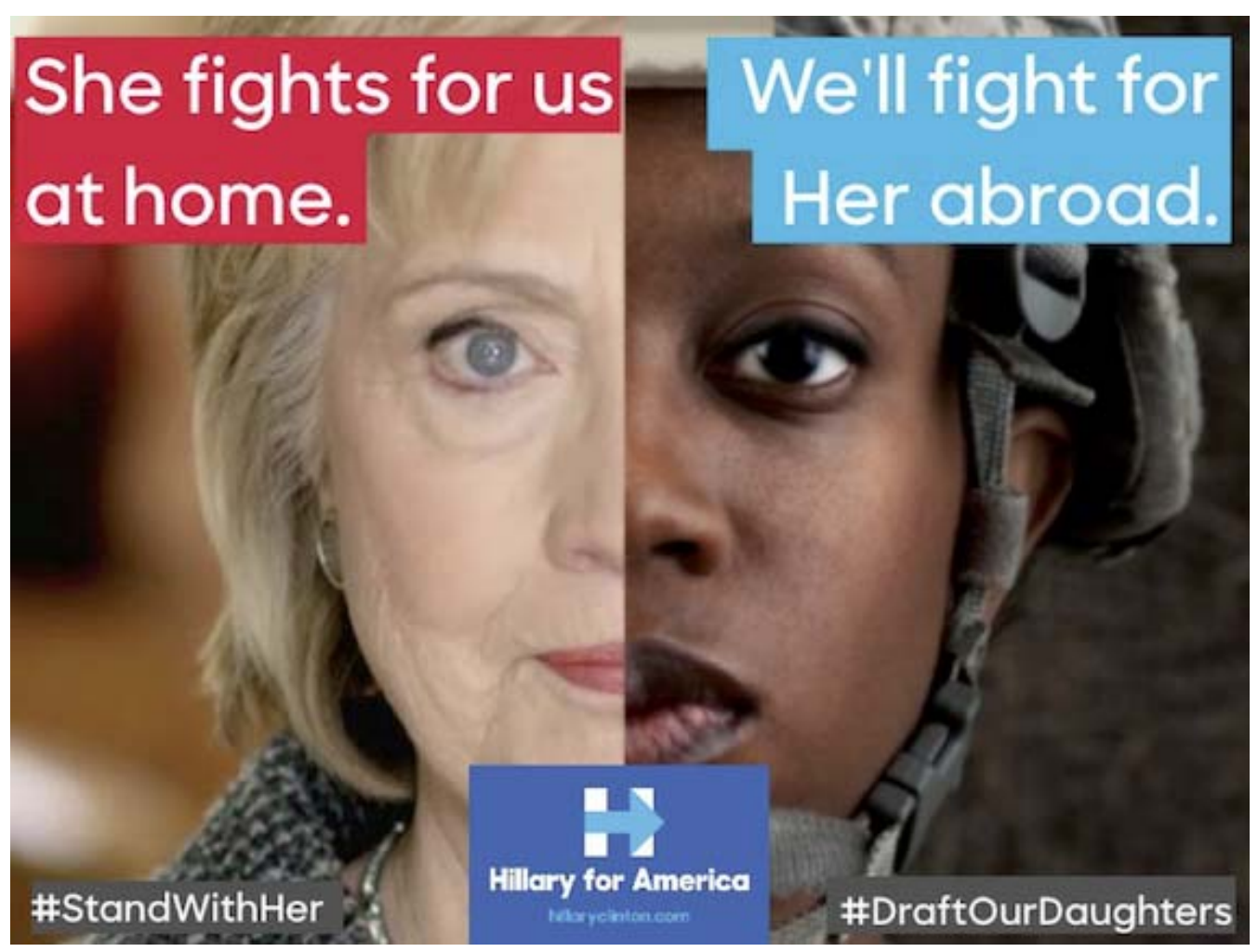

Figure 25: An example of campaign content. 


\section{Her Brother Died Fighting for his Country}

Now, it's her turn

\#ImWithHer

\#DraftOurDaughters

Figure 26: An example of campaign content. 


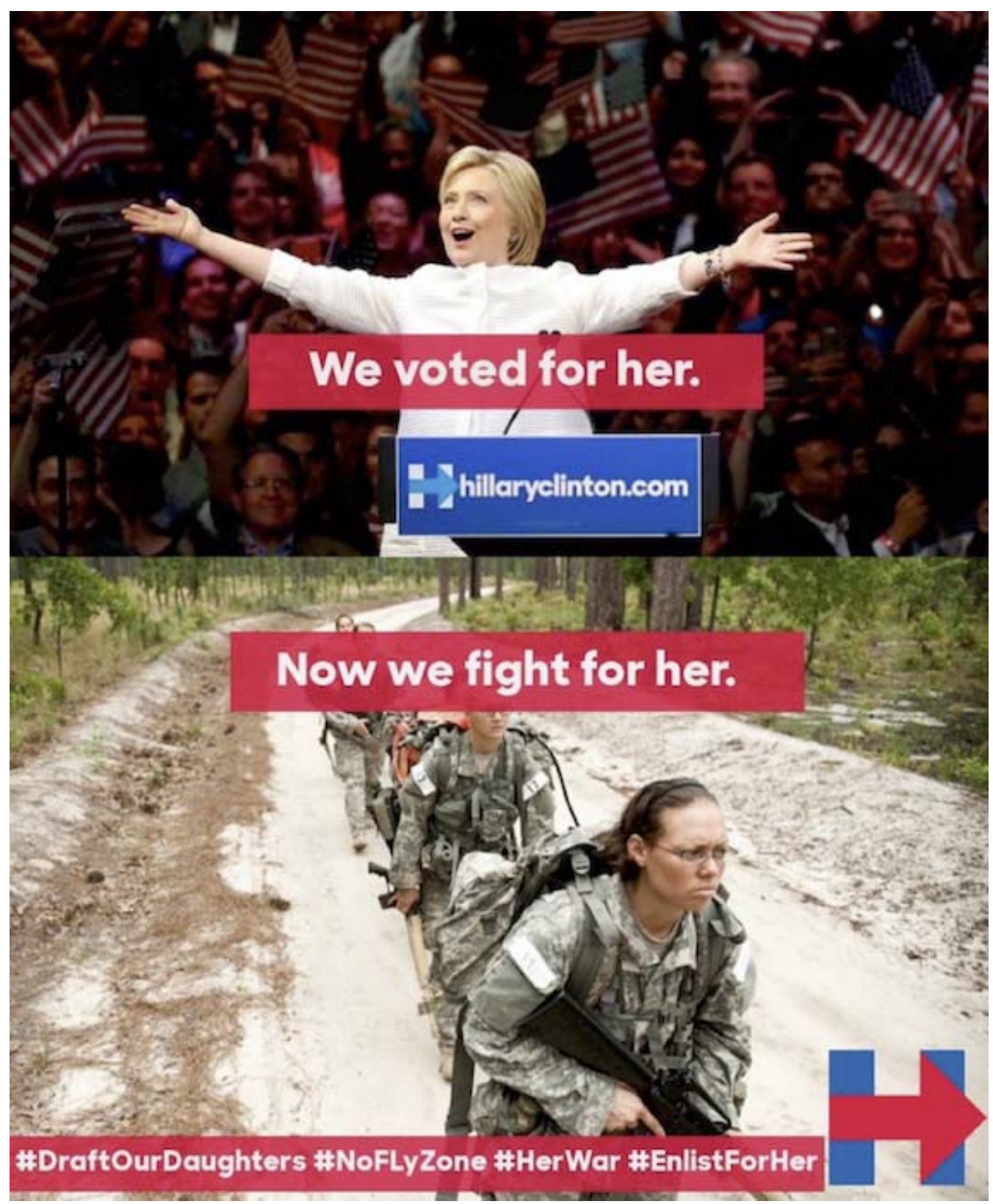

Figure 27: An example of campaign content. 


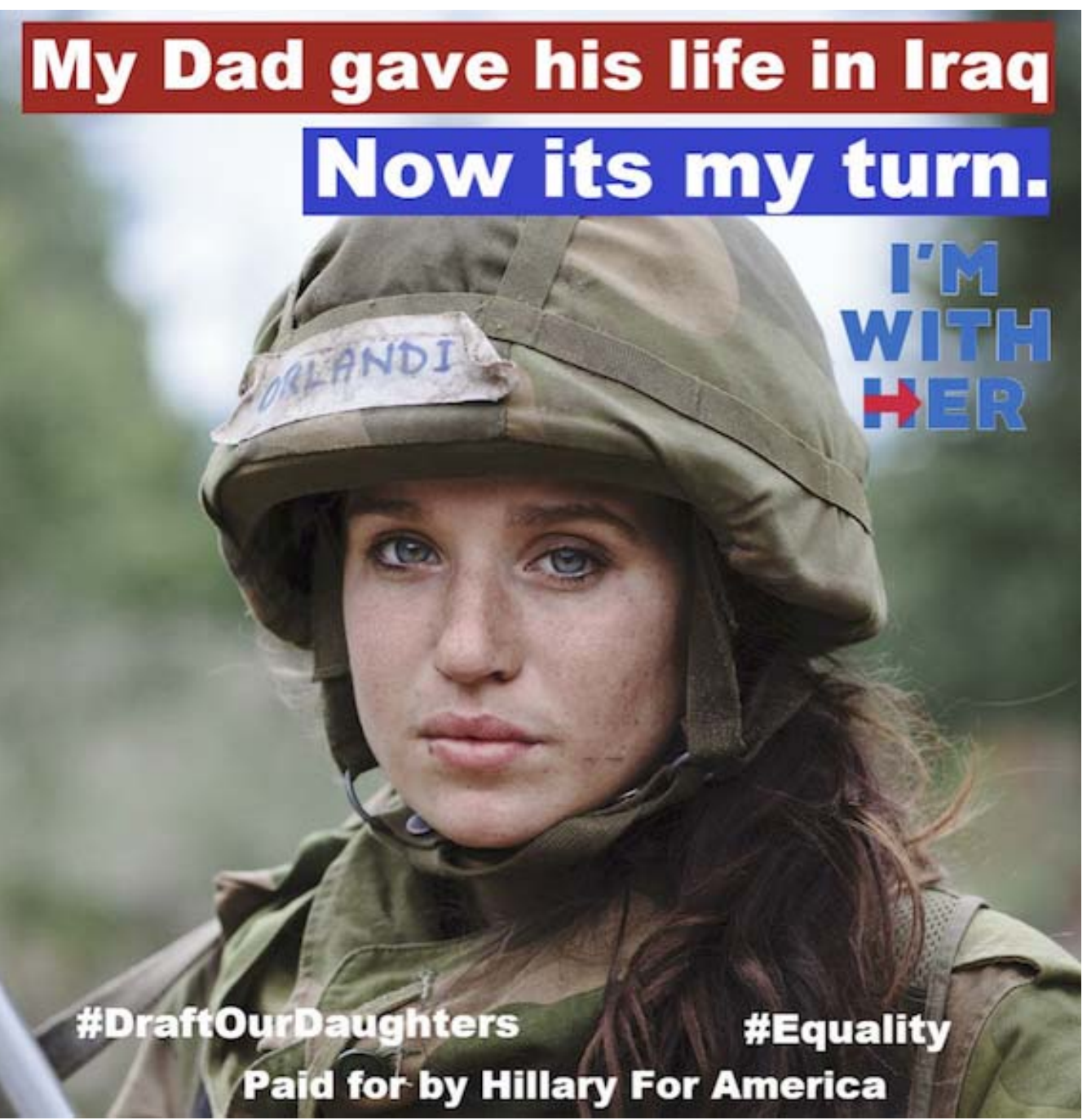

Figure 28: An example of campaign content.

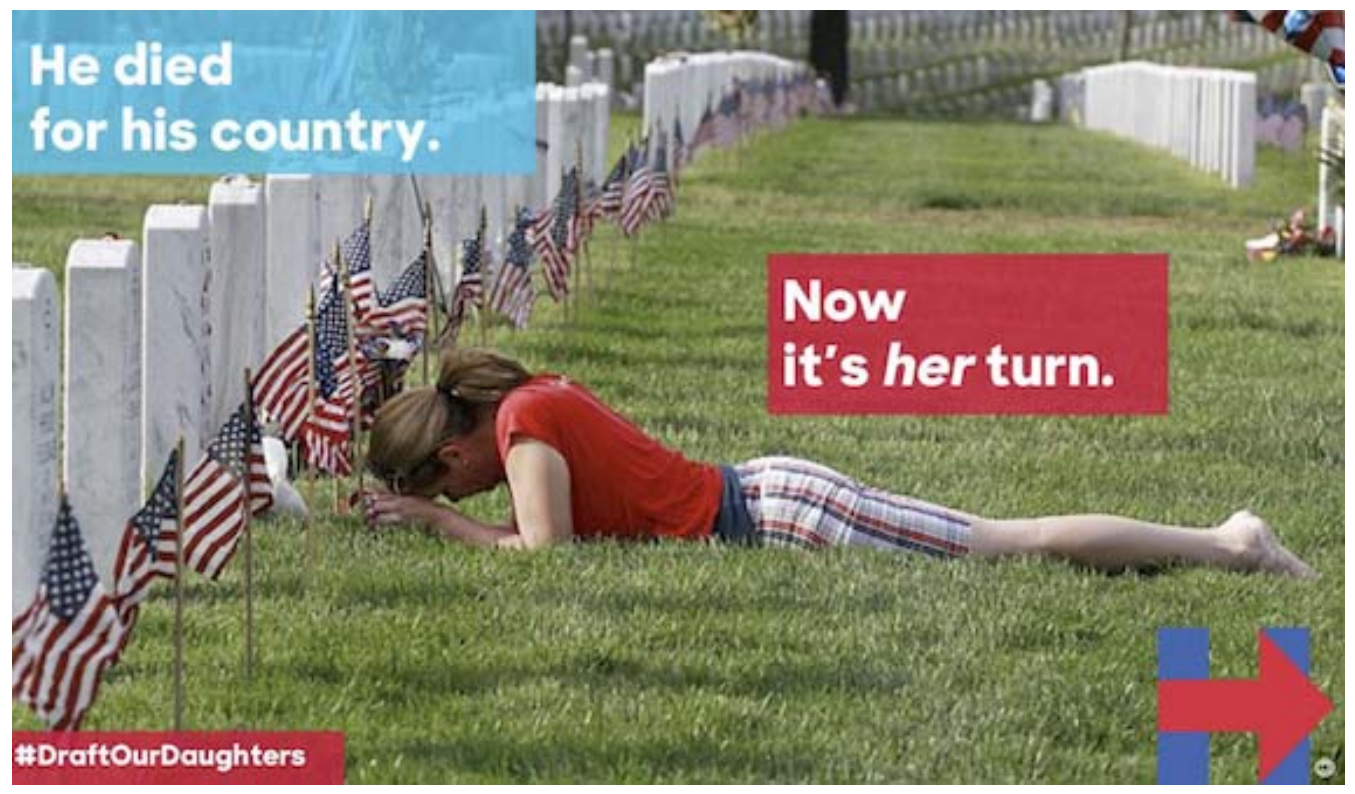

Figure 29: An example of campaign content. 


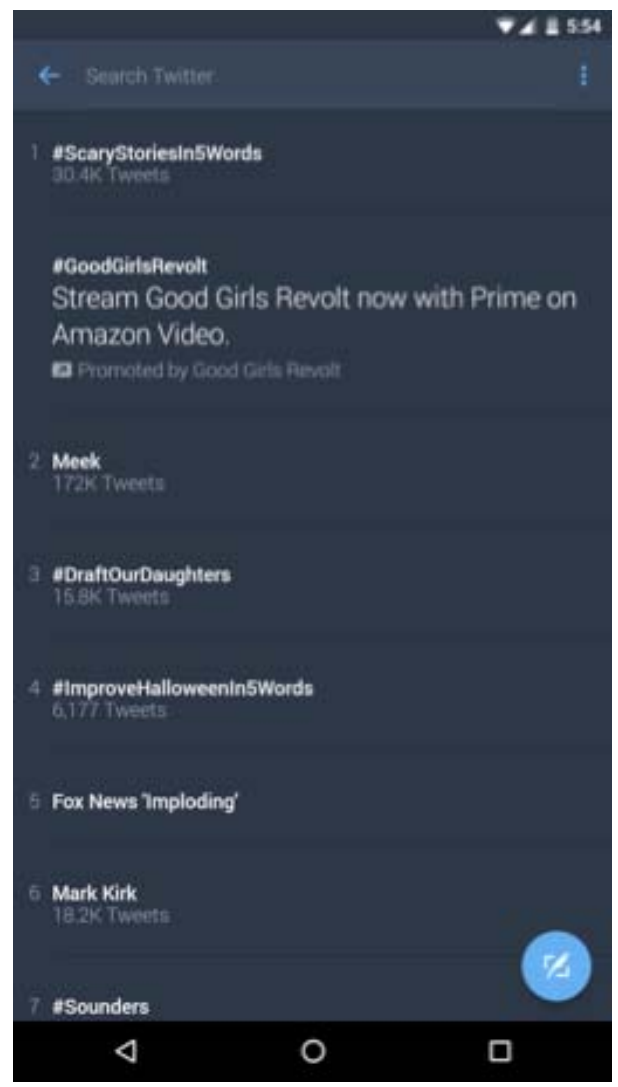

Figure 30: \#DraftOurDaughters trends on Twitter. Page archived at http://archive. is/WgLsv.

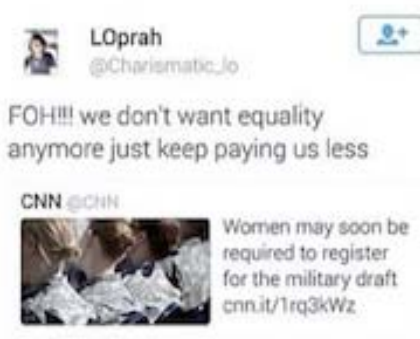

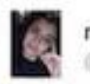

medina

Pocauhinonermedined

I suddenly don't want equality

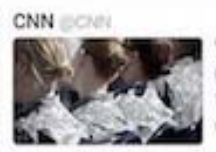

Women may soon be required to register for the military draft connit/ra3kWz

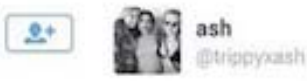

I don't want equality!!! NO

z31 cytas.07

exactly. you want equality, twittercomv meninisttweet/-

1220 PM - 15 Jun 16 bom Salinas, CI

1209 put 15 Jun 16

1 mernuter 1 ines

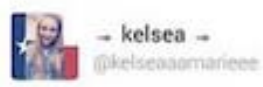

The Senate approved a bill to require women to register for the draft

1) the draft should be outlawed

2) I DONT WANT EQUAL RIGHTS LIKE

THIS

139 ps - 15 tun 10

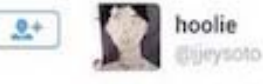

Fuck that, don't draft my ass. I didn't sign up for that portion of femininism 1255 PM 16 Jun is

Figure 31: Sample of reactions from Twitter users to \#DraftOurDaughters. 


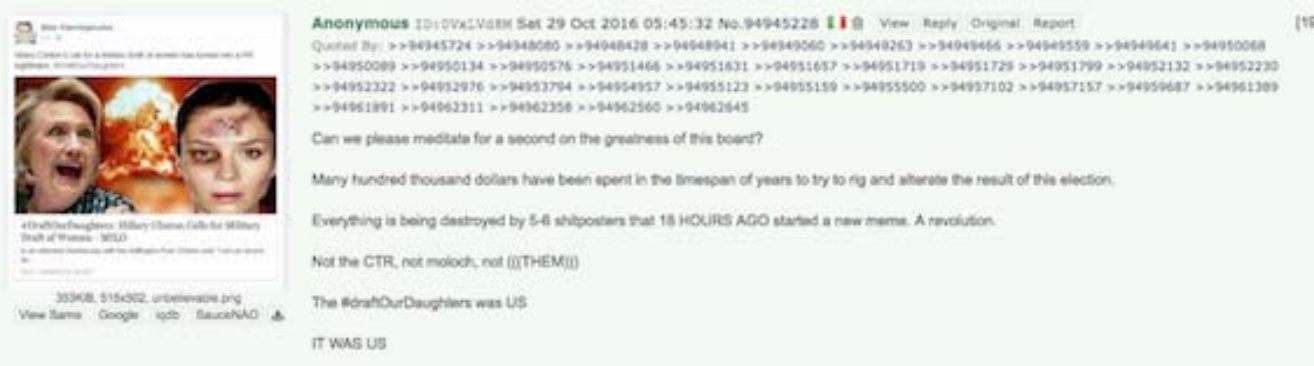

Figure 32: 4chan users reflecting on the success of the campaign. Page archived at http://archive.4plebs.org/pol/thread/94945228/.

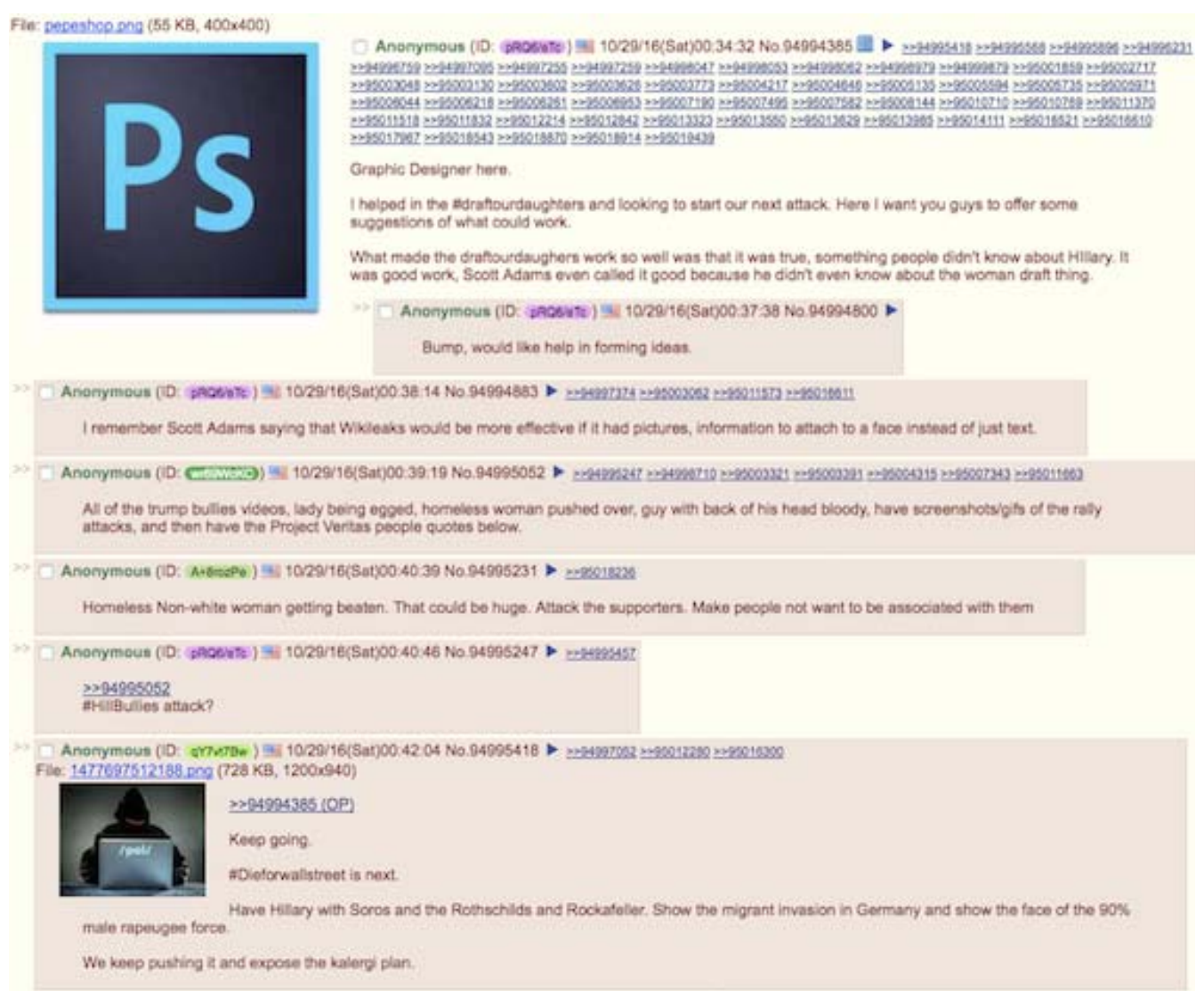

Figure 33: 4chan user identifying as a graphic designer reflecting on the success of the campaign. Page archived at http://archive.is/laJnX.

\section{Editorial history}

Received 10 December 2017; revised February 15 2018; accepted 16 February 2018.

\section{(2) $\odot \Theta^{\circ}$}

This paper is licensed under a Creative Commons Attribution-NonCommercial-ShareAlike 4.0 International License.

Swarm networks and the design process of a distributed meme warfare campaign by Travis Wall and Teodor Mitew. 
First Monday, Volume 22, Number 5 - 7 May 2018

http://firstmonday.org/ojs/index.php/fm/rt/printerFriendly/8290/7202 doi: http://dx.doi.org/10.5210/fm.v23i5.8290 